\title{
Perception-based fuzzy partitions for visual texture modelling
}

\author{
Pedro Manuel Martínez-Jiménez and Jesús Chamorro-Martínez \\ Department of Computer Science and Artificial Intelligence \\ University of Granada, Spain \\ José Manuel Soto-Hidalgo \\ Department of Computer Architecture, Electronics \\ and Electronic Technology, University of Córdoba, Spain
}

This is the peer reviewed version of the following article: Pedro Manuel Martínez-Jiménez, Jesús Chamorro-Martínez, José Manuel Soto-Hidalgo: Perceptionbased fuzzy partitions for visual texture modeling. Fuzzy Sets and Systems 337: 1-24 (2018), which has been published in final form at https://doi.org/ 10.1016/j.fss.2017.04.015. This article may be used for non-commercial purposes in accordance with Creative Commons Attribution Non-Commercial No Derivatives License (CC-BY-NC-ND license): https://creativecommons . org/licenses/by-nc-nd/4.0/legalcode.

\begin{abstract}
Visual textures in images are usually described by humans using linguistic terms related to their perceptual properties, like "very coarse", "low directional", or "high contrasted". Computational models with the ability of providing a perceptual texture characterization on the basis of these terms can be very useful in tasks like semantic description of images, content-based image retrieval using linguistic queries, or expert systems design based on low level visual features. In this paper, we address the problem of simulating the human perception of texture, obtaining linguistic labels to describe it in natural language. For this modelling, fuzzy partitions defined on the domain of some of the most representative measures of each property are employed. In order to define the fuzzy partitions, the number of linguistic labels and the parameters of the membership functions are calculated taking into account the relationship between the computational values given by the measures and the human perception of the corresponding property. The performance of each fuzzy partition is analyzed and tested using the human assessments, and a ranking of measures is obtained according to their ability to represent the perception of the property, allowing to identify the most suitable measure.
\end{abstract}

Keywords: image analysis; feature extraction; texture modelling; fuzzy partitions; linguistic labels; human perception 


\section{Introduction}

Color, texture, and shape are typically the three most used low level features for object recognition and image interpretation. Color and shape represent clear concepts for humans, and their importance is widely known in computer vision. Texture, however, is more imprecise and abstract but an equally important feature. In spite of its importance, there is not an accurate definition for the concept of texture, but some widespread intuitive ideas. Texture is described by some authors, in opposition to the idea of homogeneity, as local changes in the intensity patterns or gray tones [1]. Other authors consider texture as a set of basic items called texels (or texture primitives), arranged in a certain way. However, for humans, the most common way to describe texture is by using vague textural properties, like coarseness, directionality, contrast, line-likeness or regularity $[2,3]$, that are a more natural way to represent our perception about texture primitives. Coarseness is related to the spatial size of texels, directionality reflects whether they have a dominant orientation, contrast is related to their distinguishability, line-likeness reflects whether they have straight shapes, and regularity refers to the variation of their placement. From all of them, and according to the psychological experiments performed by Tamura et al. in [3], coarseness, contrast and directionality are considered the three most important texture properties, playing a fundamental role in human visual interpretation $[4,5,6]$.

In addition, it is natural for humans to give linguistic terms to describe the presence degree of these perceptual properties. For example, if a subject is asked about the presence degree of coarseness in the images of Figure 1, this subject would probably say that the texture shown in Figure 1(a) is "very coarse", the texture shown in Figure 1(b) is "coarse", or the texture shown in Figure 1 (c) is "very fine". Likewise, if the subject is asked about the presence degree of contrast, these textures may be perceived as "high contrasted", "medium contrasted" and "very low contrasted", respectively.

Computational models that are able to provide such kind of linguistic terms can be very useful in tasks where the most relevant information of the image lies in the presence degree of the perceptual properties of texture. In this type of tasks some interaction with subjects is usually needed, so models that describe texture as humans would are particularly interesting. For example, these models can be applied in fields such as semantic description of images [7, 8, 9], or in content-based image retrieval systems $[10,11,4]$. In this case, linguistic queries related to the presence degree of texture properties can be employed. In addition, the proposed models can be also applied in expert systems, where the information provided by the expert is related to the presence of the texture properties. For example, suppose a medical expert that, according to his/her experience, concludes that the regions with very fine and high contrasted texture in microscopic images are indicative of a certain disease. Models that are able to provide a textural description as expert would can be employed to automatically identify these areas in the images.

In this paper, we propose a perception-based fuzzy approach where each 


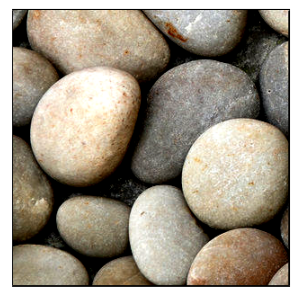

(a)

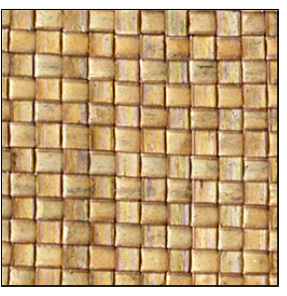

(b)

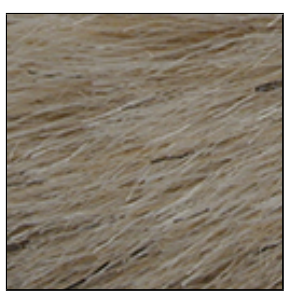

(c)

Figure 1: Examples showing the imprecision associated to the properties.

texture property will be modelled by means of a fuzzy partition defined on the domain of representative measures of the corresponding property. Because of the importance of coarseness, contrast and directionality concepts, we will focus our study on these perceptual properties (although other texture properties such as regularity, line-likeness, etc. can be easily dealt with in a similar way). In order to select the number of fuzzy sets in our partition, we analyze the ability of each measure to discriminate between different categories of the perceptual property. For this purpose, a distinguishability analysis will be applied to each measure on the basis of the human perception of the texture property. To get information about the human perception, a set of images covering different presence degrees of the properties will be used to collect, by means of polls, human assessments from a set of subjects. This way, we propose to set the number of linguistic labels used in our approach as the number of different presence degrees of the property that the measure can actually discriminate. Moreover, we propose to obtain the membership function associated to each fuzzy set by using the information given by the distinguishability analysis, obtaining a fuzzy partition adapted to the human perception of the property. This way, the presence degrees associated to the linguistic labels will match what a human would expect, providing intuitive and very useful results, as it will be shown in section 8. In addition, goodness measures are proposed in order to identify the most appropriate models to represent the properties of coarseness, contrast and directionality.

The rest of the paper is organized as follows. Section 2 describes the related work in the literature, while in section 3 a general overview of our methodology is presented, introducing some basic concepts and the notation used in the paper. After that, the different elements of the model are described in detail in the following sections; specifically, the computational measures used as reference set are summarized in section 4 , the way to obtain human assessments about the perception of the properties is faced in section 5 , the distinguishability analysis applied to the measures is detailed in section 6 , while section 7 describes the methodology employed to obtain the parameters of the proposed fuzzy partitions. In section 8 a comparative study of these models with the state of the art and some results obtained by applying them are shown. Finally, section 9 summarizes the main conclusions and future works. 


\section{Related work}

The majority of the image analysis techniques in computer vision try to model texture by means of feature vectors (that usually have very large dimensions) which have no direct relationship with the different perceptual properties. Most of these techniques are based on multiresolution analysis and scale-space theory, such as Gabor functions [12, 13, 14, 15, 16, 17] or Wavelets [18, 19, 20, 21, 22, 23], that are considered as the golden standard in the literature. In addition, general image classification and feature learning techniques can be also applied in texture analysis, such as techniques based on kernel learning [24, 25, 26, 27, $28,29]$, dictionary learning [30, 31, 32, 33] or genetic programming [34, 35, 36, 37, $38,39]$. However, none of these approaches provides a textural representation interpretable by humans. On the contrary, they are intended for comparing different textures on the basis of the similarity between feature vectors, which is a very important but completely different problem.

Although we can find in the literature some techniques that propose a texture characterization based on its perceptual properties, most of these approaches are crisp proposals $[40,41,42,43,44,3,45]$ which do not take into account the imprecision related to texture. This imprecision must be understood in the sense that it is not possible to set precise thresholds in the measure domain to give a textural interpretation (e.g. for coarseness measures, there is not an immediate way to decide whether there is a fine texture, a coarse texture or something intermediate). To face this problem, some techniques arise from the fuzzy set field [46, 47, 4, 48, 49, 50].

In the first group of approaches, each texture property is modeled as a whole by means of a unique fuzzy set, obtaining models that are able to directly represent the presence degree of the property [51, 52]. This type of approach can be very useful in classical tasks like pattern recognition. However, in other tasks, like semantic description of images or content-based image retrieval using linguistic queries, a texture characterization using linguistic terms is needed. In this case, solutions based on fuzzy partitions are more useful, because a set of linguistic labels and their corresponding membership degrees are provided. In this paper, we have focused on this second type of approaches.

In the framework of the fuzzy partition-based approaches, a mapping from low-level statistical features (the crisp measures mentioned above) to high level textural concepts is performed by defining membership functions for each textural feature. In the majority of the fuzzy techniques in the literature $[10,53$, $54,4,50,55,56]$, the measures proposed by Tamura et al. in [3] are used as reference set, and, for each texture property, a fuzzy partition with five linguistic terms is obtained. This fuzzy partition is generated through an unsupervised fuzzy clustering algorithm on the basis of the measure values obtained from an image database.

However, all these fuzzy approaches have three main drawbacks, that, to the best of our knowledge, have not been faced in the literature. The first one is related with the election of the reference set. Although there is a large number of computational measures associated with each property, in most of these 
proposals only the measures proposed by Tamura et al. in [3] are considered as reference set, without analyzing their ability to capture the corresponding property compared with other measures. The second drawback is related to the number of fuzzy set that have been proposed for the partitions. In all the approaches commented above the number of fuzzy sets are chosen arbitrarily (five fuzzy sets are usually employed), without taking into account the capability of each measure to discriminate between different categories of the corresponding property. This way, the measure may not be able to provide significantly different values for all these categories, being not possible to distinguish so many different presence degrees of the property. The third drawback of the fuzzy approaches commented above is that the parameters that define the membership functions are adjusted manually or by using a fuzzy clustering, but without considering the relationship between the measure values and the human perception of the property. This implies that the linguistic labels related to these membership functions do not necessarily match what a human would expect.

The perception-based fuzzy approach proposed in this paper allows to solve all these problems. First, different measures of each property are used as reference set and the ability of each fuzzy partition to represent the corresponding property has been analyzed, which allows to identify the most appropriate measures for coarseness, contrast and directionality properties; second, the number of fuzzy sets used in our approach has been set to the number of different categories of the perceptual property that the measure can actually discriminate, which allows to ensure that significantly different values can be provided by the measure for all the categories; and third, the membership functions associated to each fuzzy set are obtained by considering the human perception of the corresponding property, which allow to obtain presence degrees associated to the linguistic labels that match what a human would expect.

\section{Preliminaries and notations}

As mentioned in the above section, in this paper we propose to model texture properties by means of fuzzy partitions defined on the domain of computational measures. Given a texture property (coarseness, contrast, directionality, regularity, etc), let $F$ be a measure of that property and let $\mathcal{D}_{F}$ be the measure domain. The notions of fuzzy texture and fuzzy texture space are introduced as follows:

Definition 3.1. A fuzzy texture $T$ is a linguistic label whose semantics is represented by a normalized fuzzy subset of $\mathcal{D}_{F}$.

Definition 3.2. A fuzzy texture space $\Pi$ is a set of fuzzy textures that defines a partition of $\mathcal{D}_{F}$. 
From now on, let $\mathcal{P}=\left\{\right.$ fineness $^{1}$, contrast, directionality $\}$ be the set of texture properties that will be modelled in this paper and let $\mathcal{F}^{p}=\left\{F_{1}^{p}, \ldots, F_{K_{p}}^{p}\right\}$ be a set of representative computational measures of the property $p \in \mathcal{P}$. In our approach, for each property $p \in \mathcal{P}$ and each measure $F_{k}^{p} \in \mathcal{F}^{p}$ we propose to define a fuzzy partition $\Pi_{k}^{p}$ on the domain of the measure, obtaining linguistic labels associated to the texture property. We will note $N_{k}^{p}$ the number of fuzzy sets in the partition $\Pi_{k}^{p}$, and we will note $T_{k, i}^{p}$ the i-th fuzzy set in $\Pi_{k}^{p}$. The membership function $T_{k, i}^{p}(x)$ for each fuzzy set $T_{k, i}^{p}$ will be defined by using the trapezoidal function shown in Figure 2, that has the form:

$$
T_{k, i}^{p}\left(x ; a_{k, i}^{p}, b_{k, i}^{p}, c_{k, i}^{p}, d_{k, i}^{p}\right)= \begin{cases}0 & x<a_{k, i}^{p} \quad \text { or } \quad x>d_{k, i}^{p} \\ \frac{x-a_{k, i}^{p}}{b_{k, i}^{p}-a_{k, i}^{p}} & a_{k, i}^{p} \leq x \leq b_{k, i}^{p} \\ 1 & b_{k, i}^{p} \leq x \leq c_{k, i}^{p} \\ \frac{d_{k, i}^{p}-x}{d_{k, i}^{p}-c_{k, i}^{p}} & c_{k, i}^{p} \leq x \leq d_{k, i}^{p}\end{cases}
$$

In addition, a fuzzy partition in the sense of Ruspini [57] will be assumed for $\Pi_{k}^{p}$, i.e.

$$
\sum_{i=0}^{N_{k}} T_{k}^{i}(x)=1 \quad \forall x \in \mathcal{D}_{F_{k}^{p}}
$$

with $\mathcal{D}_{F_{k}^{p}}$ being the domain of the measure $F_{k}^{p}$. Some examples of this type of fuzzy partition are shown in Figure 4.

This way, three questions need to be faced in order to define the fuzzy partition $\Pi_{k}^{p}$ : (i) what reference set should be used for the fuzzy partition, (ii) how many fuzzy sets will be in the partition, and (iii) how to obtain the parameter values of the membership function for each fuzzy set. Concerning to the reference set, we will define the fuzzy partition on the domain of a given computational measure $F_{k}^{p} \in \mathcal{F}^{p}$. The measures analyzed in this paper, corresponding to fineness, contrast and directionality properties, are summarized in section 4 . All of them are automatically computed from the texture images, obtaining a real value, i.e. $\mathcal{D}_{F_{k}^{p}}=\mathbb{R}, \forall F_{k}^{p} \in \mathcal{F}^{p}$.

In order to face the other two questions, we propose to analyze the ability of each measure to distinguish between different presence degrees of the corresponding property. In this analysis, a set of multiple comparison tests is applied to each measure on the basis of the human perception of the texture property. For this purpose, two new questions need to be faced: firstly, how to obtain the data about the "human perception" of the property and, secondly, how to apply the set of multiple comparison tests in order to obtain the number of classes that can be discriminated by each measure.

\footnotetext{
${ }^{1}$ Let us remark that "coarseness" and "fineness" are opposite but related textural concepts. The advantage of modelling the concept of fineness is that the maximum presence of this property in the image is delimited by the pixel size.
} 


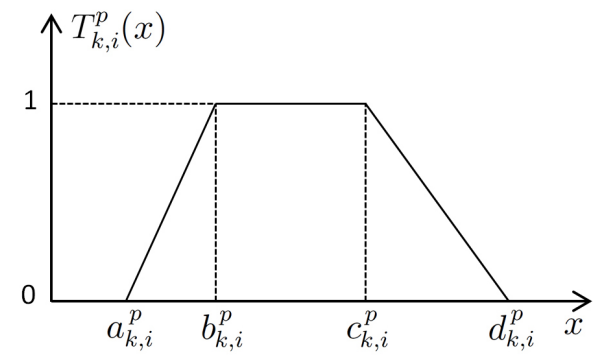

Figure 2: Trapezoidal function used for each fuzzy set $T_{k, i}^{p}$

To get information about the human perception of a property $p \in \mathcal{P}$, a set $\mathcal{I}^{p}=\left\{I_{1}^{p}, \ldots, I_{N_{p}}^{p}\right\}$ of $N_{p}$ images covering different presence degrees of this property is gathered. These images are arranged, by means of a poll, into different classes with an associated presence degree of the property. The description of the texture image set and the way to obtain this initial classification are detailed in section 5 . In order to estimate the number of classes that can be discriminated by each measure $F_{k}^{p} \in \mathcal{F}^{p}$, an iterative algorithm based on a set of multiple comparison tests is applied to the measure values calculated for the images in $\mathcal{I}^{p}$. This algorithm starts with the initial classification of these images given by the polled subjects, and iteratively joins clusters until a partition in which all classes are distinguishable is achieved. The description of this distinguishability analysis algorithm is detailed in section 6 .

Finally, given a measure $F_{k}^{p} \in \mathcal{F}^{p}$, we propose to set the number of fuzzy sets $N_{k}^{p}$ in the partition $\Pi_{k}^{p}$ to the number of classes that can be discriminated by this measure. In addition, the parameter values of the membership function $T_{k, i}^{p}(x)$ for each fuzzy set $T_{k, i}^{p}$ are obtained by using the information given by the representative values and the confidence intervals associated to these classes. The detailed description of the methodology employed to obtain these parameters is presented in section 7 .

\section{Computational measures: the reference set}

There are many measures in the literature that, given an image, capture the presence of a textural property in the sense that the greater the value given by the measure, the greater (lower) the presence of the property. In this study, we propose to use the computational measures employed in our previous work [52], corresponding to fineness, contrast and directionality properties. In this section, these measures are summarized.

\subsection{Fineness measures}

Among all the perceptual texture properties, the coarseness-fineness is the most popular one, being considered as the most fundamental feature in texture anal- 


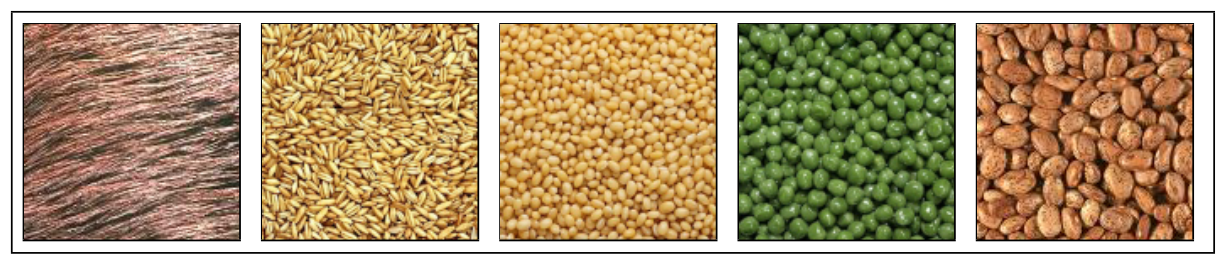

(a)

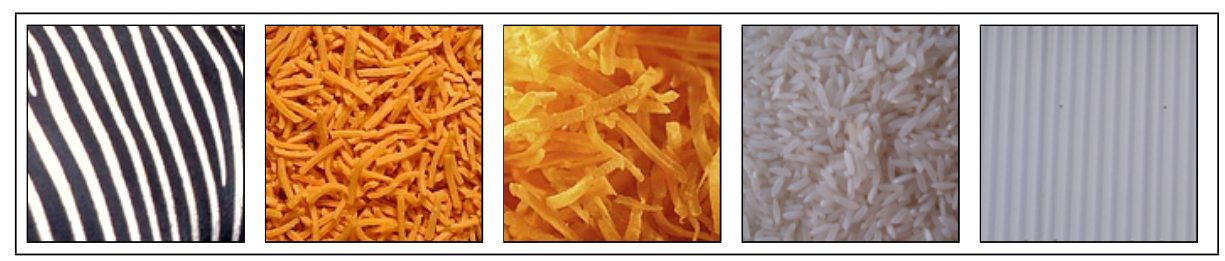

(b)

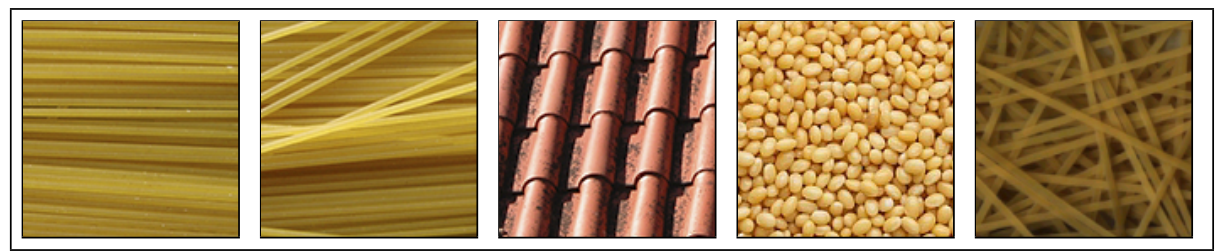

(c)

Figure 3: Some examples of the texture images used in the poll corresponding to the properties of fineness (a), contrast (b) and directionality (c). These images are in decreasing order according to the presence degree of the corresponding property.

ysis by some authors [4]. In fact, the presence of fineness is usually associated to the presence of texture (from this point of view, texture is defined as local variations against the idea of homogeneity). In this sense, a fine texture contains small texture primitives with large gray tone differences between neighbor pixels (e.g. the first image of Figure 3(a)), whereas a coarse texture corresponds to larger primitives formed by several pixels (e.g. the last image of Figure 3(a)).

In this paper, we have initially considered the 17 fineness measures analyzed in our previous work [58]. These measures can be classified into 3 groups according to the strategy used to quantify the coarseness of the texture image. The first group includes those measures that try to estimate directly the size of the texels by analyzing the pixels of the image. In this group we can find the measure defined by Abbadeni et al. in [59], the measure proposed by Tamura et al. in [3], the Edge Density (ED measure), that is calculated as the percentage of pixels which are an edge in the image, and the Fractal Dimension (FD measure) defined by Mandelbrot in [60], that is estimated by following the blanket method introduced by Peleg in [61]. 
The second group includes the measures obtained by applying statistics over matrices that collect information about the relationships between the gray level of each pixel and their neighbours. The measures of Haralick [41], that are based on the GLCM matrix [62], are placed in this group. In particular, 6 coarseness measures are obtained by applying the statistics contrast, correlation, entropy, local homogeneity, variance and uniformity over this matrix. This group also includes the measure defined by Amadasun in [63], the Short Run Emphasis (SRE measure) given by Galloway in [64], the Small Number Emphasis (SNE measure) defined by Sun et al. in [65], the Distribution of Gray Level Difference (DGD measure) proposed by Kim et al. in [66], and the measure defined by Weszka et al. in [67].

The third group is composed of two measures that are based on the Fourier power spectrum of the image. The first one is the measure used by Newsam in [68], computed as the average of power spectrum over ring-shaped regions centered at the origin, and the second one is the first moment of the power spectrum (FMPS measure), obtained by computing the mean value of Fourier power spectrum of the image [69].

However, according to the study performed in [58], some of the above measures have an unsuitable behavior. The measures of Newsam, FMPS, Entropy and uniformity are size dependent, i.e. the values given by these measures are affected by the window size. In addition, the Variance measure does not provide a representative information about the perception of fineness. Thus, these five measures are rejected and they will not be taken into account in the following, focusing our study on the other 12 measures, that are listed in the first column of Table 1(a). Besides the independence with respect to the image size and the ability to provide information about the fineness perception, other interesting properties for texture analysis are also fulfilled by the proposed measures. As it is shown in [58], they are robust against changes in the image characteristic, like brightness and contrast, and they have a suitable behavior regarding the presence of noise in the image.

\subsection{Contrast measures}

The contrast property reflects the clarity with which texture primitives are distinguishable. In this sense, a well contrasted texture contains primitives that are clearly visible and separable (e.g. the first image of Figure 3(b)), whereas a low contrasted texture has a poor distinguishability between texels (e.g. the last image of Figure 3(b)). Notice that this property refers to grayscale images, and it should not be confused with the contrast related to color images.

In this paper, we propose to use 4 of the most used contrast measures in the literature. Two of them try to estimate directly the contrast between texels by analyzing the pixels of the image. The first one is the measure defined by Tamura et al. in [3], which takes into account both the dynamic range of gray levels in the image and the kurtosis of their distribution. The second one is the contrast measure defined by Abbadeni in [59], which is based on the autocovariance function. 
The other two measures are obtained by applying statistics over matrices that collect information about the relationships between the gray level of each pixel and their neighbours. The first one is the contrast statistic proposed by Haralick et al. in [41], which is obtained from GLCM matrices. The second one is the contrast measure proposed by Amadasun and King in [63], which takes into account both global statistics (as the dynamic range of gray levels in the image) and local statistics calculated from the Neighbourhood Gray-Tone Difference Matrix.

\subsection{Directionality measures}

The directionality property is related to the presence of a dominant orientation in texture primitives, and it depends on two factors: (i) the shape of texture primitives individually, and (ii) their placement rule in the image. In this sense, a directional texture contains primitives that have a dominant dimension, i.e. texels with an elongated shape, and, in addition, this primitives are arranged in the same orientation (e.g. the first image of Figure 3(c)). The directionality presence decreases as the orientation of all texels does not match, even if they have an elongated shape (e.g. the second image of Figure 3(c)), or as the shape of texels is less elongated, even if they are arranged in the same orientation (e.g. the third image of Figure 3(c)). The absence of directionality (omnidirectional texture) is associated to texture primitives that do not have a dominant dimension in their shape (e.g. the fourth image of Figure 3(c)) or such that their arrangement does not have a dominant orientation (e.g. the last image of Figure 3(c)).

In this paper, we will use 3 of the most known directionality measures from the literature. The first one is the directionality measure defined by Tamura et al. in [3], which is based on the computation of an histogram of local edge probabilities against their directional angle. The second one is the directionality measure proposed by Noureddine Abbadeni in [59], which is also based on the analysis of directional angles, but considering the local edges obtained from the autocovariance function, instead of the local edges of the original image. The last measure is the directionality statistic computed from the Fourier power spectrum of the image [67,68], which analyzes the energy distribution in wedgeshaped regions of the frequency domain.

\section{$5 \quad$ Study of the perception of properties}

In this section, we will describe the methodology proposed to classify the images in the set $\mathcal{I}^{p}$ according to the human perception of the corresponding texture property. For this purpose, first a image set $\mathcal{I}^{p}$ is selected for each property $p \in \mathcal{P}$ (section 5.1). After that, a poll is designed for assigning images into different classes with an associated presence degree of the property (section 5.2). 


\subsection{The texture image set}

For each property $p \in \mathcal{P}$, a set $\mathcal{I}^{p}=\left\{I_{1}^{p}, \ldots, I_{N_{p}}^{p}\right\}$ of $N_{p}=80$ images representing examples of this property has been selected. Figure 3 shows some images extracted from the set $\mathcal{I}^{p}$ corresponding to the properties of fineness (Figure $3(\mathrm{a})$ ), contrast (Figure 3(b)) and directionality (Figure 3(c)). Each set has been selected satisfying the following conditions:

- It covers the different presence degrees of the property.

- The number of images for each presence degree is representative enough.

- Each image shows, as far as possible, just one presence degree of the property.

Due to the third condition, each image $I_{i}^{p} \in \mathcal{I}^{p}$ can be viewed as "homogeneous" respect to the presence degree of the corresponding property, i.e., if we select two random windows (with a dimension which does not "break" the original texture primitives and structure), the perceived presence of the property will be similar for each window (and also with respect to the original image). In other words, we can see each image $I_{i}^{p} \in \mathcal{I}^{p}$ as a set of lower dimension images (sub-images) with the same presence degree of the original one. This will be very useful in our approach, because we can have a larger number of samples in the distinguishability analysis without extending the number of images that the subjects have to classify in the poll. From now on, we will note as $\mathcal{I}_{\mathcal{W}}^{p}=\left\{I_{i, w}^{p}, i=1, \ldots, N_{p} ; w=1, \ldots, W_{p}\right\}$ the set of all the sub-images extracted from $\mathcal{I}^{p}$, where $I_{i, w}^{p}$ is the $w$-th sub-image of $I_{i}^{p}$ and $W_{p}$ is the number of sub-images considered for each image.

\subsection{The poll}

Given the image set $\mathcal{I}^{p}$, the next step is to obtain an initial classification of the images according to the human perception of the property $p \in \mathcal{P}$. For this purpose, a poll has been designed allowing to assign the images of $\mathcal{I}^{p}$ to different classes, where each class has an associated presence degree of the property. In our proposal, an example image which represents the corresponding presence degree is associated to each class. In order to make this classification easier for subjects, they were first asked to sort the images of $\mathcal{I}^{p}$ according to their presence degree of the texture property.

From now on, let $R^{p}$ be the number of classes that have been considered in the poll for the property $p \in \mathcal{P}$. One of the classes is associated to the presence degree 1 of this property. In our proposal, traditional examples used in the literature to define very fine, high contrasted and very directional textures have been considered for this class [70]. An example that represents this presence degree for each property is shown in the first image of figures 3(a), 3(b) and 3 (c), respectively. Another of the classes considered in the poll represents the presence degree of 0 of the property. In this case, again, traditional examples 
used in the literature to define very coarse, very low contrasted and very nondirectional textures has been considered for this class. The last image of figures $3(\mathrm{a})$ and 3(b) shows an example of this presence degree for fineness and contrast properties, respectively. In the case of directionality, the presence degree of 0 is associated to texture primitives that do not have a dominant dimension or such that their arrangement does not have a dominant orientation, as it is shown in the last two images of Figure 3(c).

The rest of classes represent presence degrees of the property between 0 and 1. In the case of fineness, nine classes have been regarded, considering gradual variations in the size of texture primitives, five of which are shown in the sample images of Figure 3(a). It should be noticed that these images are in decreasing order according to the presence degree of the fineness concept. In the case of contrast, five classes have been considered, taking into account gray level differences in texel edges in order to scale their contrast between the classes corresponding to degrees 1 and 0 . The five representative images used in the poll are shown in Figure 3(b). In the case of directionality, six classes have been regarded, considering gradual variations in texels orientation or/and shape, five of which are shown in Figure 3(c).

This way, an initial classification of the image set $\mathcal{I}^{p}$ into the $R^{p}$ classes used in the poll has been obtained, where each class will contain the images assigned to it by the majority of the 20 subjects who have participated in the poll. As commented above, thanks to the "homogeneity" in the presence degree of the property, all the sub-images of $I_{i}^{p}$ should be classified in the same class as $I_{i}^{p}$. Thus, a partition ${ }^{2}$ of $\mathcal{I}_{\mathcal{W}}^{p}$ in $R^{p}$ classes is obtained, that we will note Part $_{\mathcal{I}_{\mathcal{W}}^{p}}=\left\{C_{1}, C_{2}, \ldots, C_{R^{p}}\right\}$.

In this paper, for each image $I_{i}^{p} \in \mathcal{I}^{p}, W=200$ sub-images of size $32 \times 32$ have been considered ${ }^{3}$, so $\mathcal{I}_{\mathcal{W}}^{p}$ is formed by 16000 sub-images for each property $p \in \mathcal{P}$. We propose to randomly select $75 \%$ of them for the distinguishability analysis, and the rest will be used for testing the obtained fuzzy partitions. From now on, we will note $\mathcal{I}_{\mathcal{W}}^{p \text {,analysis }}$ the set of the selected sub-images from $\mathcal{I}_{\mathcal{W}}$, and we will note $\mathcal{I}_{\mathcal{W}}^{p \text {,test }}$ the set of the rest of sub-images. The partitions of these two sets in the $R^{p}$ classes, noted as Part ${ }_{\mathcal{I}_{\mathcal{W}}^{\text {panalysis }}}$ and Part $_{\mathcal{I}_{\mathcal{W}}^{\text {, test }}}$ respectively, are obtained immediately from $\operatorname{Part}_{\mathcal{I}_{\mathcal{W}}^{p}}$.

\section{Distinguishability analysis of the measures}

At this point, the aim is to obtain the number of classes that can be discriminated by a given measure $F_{k}^{p} \in \mathcal{F}^{p}$ of a property $p \in \mathcal{P}$. To do this, we propose to analyze each measure by following the iterative method detailed in the Algorithm 1. This algorithm starts with a initial partition $\operatorname{Part}_{p, k}^{0}$ (that in

\footnotetext{
${ }^{2}$ Note that this is not a fuzzy partition but a crisp one.

${ }^{3}$ As mentioned in Section 4 , the measures used in this study are not size dependent. Therefore, the models obtained by means of the fitting process don't depend on the window size. Sub-images smaller than $32 \times 32$ are not considered because they would break texture primitives.
} 


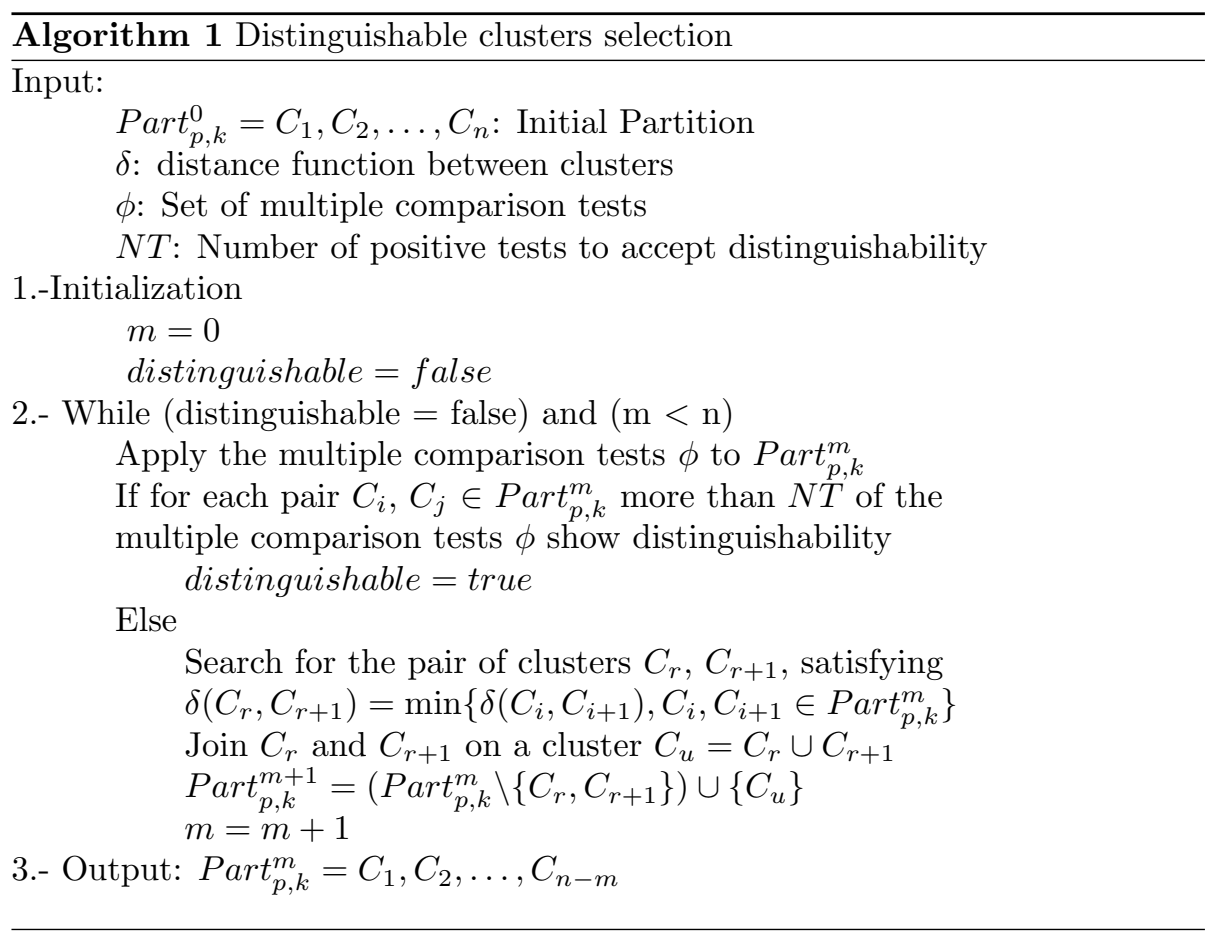

our approach is $\operatorname{Part}_{\mathcal{I}_{\mathcal{W}}^{\text {p,analysis }}}$ ) and iteratively joins clusters until a partition in which all classes are distinguishable is achieved. For this purpose, in each iteration a set of multiple comparison tests $\phi$ is applied to the corresponding partition in order to analyze the distinguishability of the classes. If the number of positive tests is less than $N T$ for any pair of classes, a distance between classes is computed by means of a function $\delta$, joining the pair with the minimum distance. The obtained partition is analyzed in the next iteration and this process continues until the number of positive tests is equal or greater than $N T$ for all the pair of classes. In this case, we consider that all the classes in the partition are distinguishable, obtaining the number of classes that can be discriminated by the analyzed measure. Note that the proposed iterative method follows a classical agglomerative hierarchical clustering algorithm, where the distance metric is given by the function $\delta$, but in this case the merging process stops when all the clusters are distinguishable instead of building all the hierarchy.

In our proposal, the following input parameters for Algorithm 1 have been selected:

- $\phi$ is a set of 5 multiple comparison tests listed below [71]:

- Scheffé test

- Bonferroni test 
Table 1: Result obtained from the distinguishability analysis for the properties of fineness (a), contrast (b) and directionality (c).

\begin{tabular}{|l|c|c|c|c|c|c|c|}
\hline $\begin{array}{l}\text { Fineness } \\
\text { measure }\end{array}$ & $N_{k}^{p}$ & $\begin{array}{c}\text { Grouped } \\
\text { classes }\end{array}$ & $r_{k, 5}^{p} \pm \Psi_{k, 5}^{p}$ & $r_{k, 4}^{p} \pm \Psi_{k, 4}^{p}$ & $r_{k, 3}^{p} \pm \Psi_{k, 3}^{p}$ & $r_{k, 2}^{p} \pm \Psi_{k, 2}^{p}$ & $r_{k, 1}^{p} \pm \Psi_{k, 1}^{p}$ \\
\hline Correlation & 5 & $\{1,2-4,5-6,7-8,9\}$ & $0.1145 \pm 0.0378$ & $0.4066 \pm 0.0282$ & $0.4988 \pm 0.0238$ & $0.6112 \pm 0.0135$ & $0.7747 \pm 0.0202$ \\
ED & 5 & $\{1,2,3-5,6-8,9\}$ & $0.3531 \pm 0.0091$ & $0.2914 \pm 0.0067$ & $0.2707 \pm 0.0070$ & $0.2462 \pm 0.0084$ & $0.1796 \pm 0.0066$ \\
Amadasun & 4 & $\{1,2-4,5-8,9\}$ & - & $0.1936 \pm 0.0073$ & $0.3439 \pm 0.0113$ & $0.4165 \pm 0.0102$ & $0.8000 \pm 0.0571$ \\
Contrast & 4 & $\{1,2-5,6-8,9\}$ & - & $3325 \pm 282.3$ & $2572 \pm 308$ & $1866 \pm 98.83$ & $796.6 \pm 132.7$ \\
FD & 4 & $\{1,2,3-8,9\}$ & - & $3.3857 \pm 0.0360$ & $3.1640 \pm 0.0282$ & $2.9763 \pm 0.0518$ & $2.5713 \pm 0.0394$ \\
Tamura & 4 & $\{1,2-6,7-8,9\}$ & - & $1.540 \pm 0.0634$ & $1.864 \pm 0.0722$ & $2.125 \pm 0.0420$ & $3.045 \pm 0766$ \\
Weszka & 4 & $\{1,2-6,7-8,9\}$ & - & $0.153 \pm 0.0064$ & $0.113 \pm 0.0093$ & $0.099 \pm 0.0036$ & $0.051 \pm 0.0041$ \\
Abbadeni & 3 & $\{1,2-8,9\}$ & - & - & $5.9054 \pm 0.3070$ & $10.791 \pm 0.2814$ & $25.899 \pm 2.7423$ \\
DGD & 3 & $\{1,2-8,9\}$ & - & - & $0.0237 \pm 0.0014$ & $0.0419 \pm 0.0017$ & $0.0947 \pm 0.0076$ \\
LH & 3 & $\{1,2-8,9\}$ & - & - & $0.0216 \pm 0.0013$ & $0.0514 \pm 0.0026$ & $0.1285 \pm 0.0112$ \\
SNE & 3 & $\{1,2-8,9\}$ & - & - & $0.8781 \pm 0.0188$ & $0.7752 \pm 0.0092$ & $0.5668 \pm 0.0251$ \\
SRE & 3 & $\{1,2-8,9\}$ & - & - & $0.9962 \pm 0.0004$ & $0.9885 \pm 0.0007$ & $0.9665 \pm 0.0037$ \\
\hline
\end{tabular}

(a)

\begin{tabular}{|l|c|c|c|c|c|c|c|}
\hline $\begin{array}{l}\text { Contrast } \\
\text { measure }\end{array}$ & $N_{k}^{p}$ & $\begin{array}{c}\text { Grouped } \\
\text { classes }\end{array}$ & $r_{k, 5}^{p} \pm \Psi_{k, 5}^{p}$ & $r_{k, 4}^{p} \pm \Psi_{k, 4}^{p}$ & $r_{k, 3}^{p} \pm \Psi_{k, 3}^{p}$ & $r_{k, 2}^{p} \pm \Psi_{k, 2}^{p}$ & $r_{k, 1}^{p} \pm \Psi_{k, 1}^{p}$ \\
\hline Tamura & 5 & $\{1,2,3,4,5\}$ & $0.1294 \pm 0.0116$ & $0.2398 \pm 0.0109$ & $0.4590 \pm 0.0184$ & $0.6508 \pm 0.0225$ & $1.2379 \pm 0.0286$ \\
Amadasun & 5 & $\{1,2,3,4,5\}$ & $0.2727 \pm 0.0284$ & $0.4449 \pm 0.0397$ & $0.8362 \pm 0.0665$ & $1.1792 \pm 0.0818$ & $2.6313 \pm 0.1454$ \\
Abbadeni & 4 & $\{1,2,3-4,5\}$ & - & $0.5343 \pm 0.0392$ & $0.9796 \pm 0.0586$ & $1.7591 \pm 0.0936$ & $3.4674 \pm 0.2687$ \\
Haralick & 4 & $\{1-2,3,4,5\}$ & - & $0.5375 \pm 0.0383$ & $0.9735 \pm 0.0755$ & $1.6307 \pm 0.1633$ & $3.0789 \pm 0.2472$ \\
\hline
\end{tabular}

(b)

\begin{tabular}{|l|c|c|c|c|c|c|c|}
\hline $\begin{array}{l}\text { Directionality } \\
\text { measure }\end{array}$ & $N_{k}^{p}$ & $\begin{array}{c}\text { Grouped } \\
\text { classes }\end{array}$ & $r_{k, 5}^{p} \pm \Psi_{k, 5}^{p}$ & $r_{k, 4}^{p} \pm \Psi_{k, 4}^{p}$ & $r_{k, 3}^{p} \pm \Psi_{k, 3}^{p}$ & $r_{k, 2}^{p} \pm \Psi_{k, 2}^{p}$ & $r_{k, 1}^{p} \pm \Psi_{k, 1}^{p}$ \\
\hline Tamura & 5 & $\{1,2-3,4,5,6\}$ & $0.8423 \pm 0.0049$ & $0.9070 \pm 0.0043$ & $0.9398 \pm 0.0047$ & $0.9643 \pm 0.0039$ & $0.9846 \pm 0.0038$ \\
Fourier & 5 & $\{1,2,3-4,5,6\}$ & $0.0535 \pm 0.0060$ & $0.1283 \pm 0.0139$ & $0.2099 \pm 0.0131$ & $0.2778 \pm 0.0189$ & $0.3672 \pm 0.0198$ \\
Abbadeni & 4 & $\{1,2-3,4-5,6\}$ & - & $0.2516 \pm 0.0391$ & $0.6380 \pm 0.0298$ & $0.8026 \pm 0.0189$ & $0.9638 \pm 0.0077$ \\
\hline
\end{tabular}

(c)

- Duncan test

- Tukey's least significant difference test

- Tukey's honestly significant difference test

- The number of positive tests to accept distinguishability will be fixed to $N T=3$.

- The distance function $\delta$ is defined as $\delta=1 / p$, where $p$ is the $p$-value computed from the analysis of variance (ANOVA) [72, 73] of the involved classes: the more overlapped the classes, the shorter the obtained distance.

From now on, we shall note as $\Upsilon_{k}^{p}=C_{k, 1}^{p}, C_{k, 2}^{p}, \ldots, C_{k, N_{k}^{p}}^{p}$ the $N_{k}^{p}$ classes that can be discriminated by the measure $F_{k}^{p} \in \mathcal{F}^{p}$ associated to the property $p \in \mathcal{P}$. For each class $C_{k, i}^{p}$, we will note as $r_{k, i}^{p}$ the class representative value. In this paper, we propose to compute $r_{k, i}^{p}$ as the mean of the measure values in the class $C_{k, i}^{p}$.

Table 1 shows the results obtained by applying the proposed algorithm with the different measures considered in this paper. The second column of this 
table shows the $N_{k}^{p}$ classes that can discriminate each measure and the third column shows how the initial classes have been grouped. The columns from fourth to eighth show the representative values $r_{k, i}^{p}$ associated to each cluster. It can be noticed that, for the property of fineness, the measures of Correlation and $E D$ are able to discriminate five different classes of this property, the measures of Amadasun, Contrast, FD, Tamura and Weszka can discriminate four classes, while the other measures are not able to discriminate more than three classes of fineness. In the case of the contrast property, we can see that the measures of Tamura and Amadasun can distinguish five different classes of this property, while the measures of Abbadeni and Haralick are able to discriminate four. Finally, with regard to the directionality property, five classes can be distinguishable for the measures of Tamura and Fourier, and four for the measure of Abbadeni.

\section{$7 \quad$ The fuzzy partitions}

In this section we will deal with the problem of obtaining, for each fuzzy set $T_{k, i}^{p}$ in the partition $\Pi_{k}^{p}$, the parameters of the membership function $T_{k, i}^{p}(x)$, which is defined by using the trapezoidal function given by equation (1). As it was commented in section 3 , the number of fuzzy sets $N_{k}^{p}$ of the partition will be given by the number of categories that each measure can discriminate (shown in Table 1). In addition, we propose to obtain the parameters of the corresponding membership functions on the basis of the information given by the multiple comparison tests used in the previous section.

As it is known, in these tests confidence intervals around the representative value of each class are calculated, being accomplished that these intervals do not overlap for distinguishable classes. The interpretation of the confidence interval associated to a class is that all values belonging to them are considered as plausible values for the estimated mean of this class. These confidence intervals are defined by the following parameter

$$
\Psi_{k, i}^{p}=1.96 \bar{\sigma}_{k, i}^{p} / \sqrt{\left\|C_{k, i}^{p}\right\|}
$$

with $\bar{\sigma}_{k, i}^{p}$ and $\left\|C_{k, i}^{p}\right\|$ being the estimated standard deviation and the cardinality of the class $C_{k, i}^{p}$, respectively. Table 1 shows the values $\Psi_{k, i}^{p}$ for each class associated to each measure $F_{k}^{p} \in \mathcal{F}^{p}$.

Based on this idea, we propose to set the center of the kernel of the membership function $T_{k, i}^{p}(x)$ as the representative value $r_{k, i}^{p}$ of the corresponding i-th class, using the information of the confidence interval to obtain the value of the parameters $b_{k, i}^{p}$ and $c_{k, i}^{p}$ :

$$
b_{k, i}^{p}=r_{k, i}^{p}-\Psi_{k, i}^{p} \quad c_{k, i}^{p}=r_{k, i}^{p}+\Psi_{k, i}^{p}
$$

Since a fuzzy partition in the sense of Ruspini is proposed, the value of the parameters $a_{k, i}^{p}$ and $d_{k, i}^{p}$ is obtained as 
Table 2: Performance evaluation and goodness measure of the proposed partitions for the properties of fineness (a), contrast (b) and directionality (c).

\begin{tabular}{|c|c|c|c|c|c|c|c|c|c|}
\hline $\begin{array}{l}\text { Fineness } \\
\text { measure }\end{array}$ & precision & recall & $f_{k}^{p}$ & $g_{k}^{p}$ & $\begin{array}{l}\text { Contrast } \\
\text { measure }\end{array}$ & precision & recall & $f_{k}^{p}$ & $g_{k}^{p}$ \\
\hline Amadasun & 0.7486 & 0.7744 & 0.7613 & 0.6852 & Tamura & 0.9109 & 0.8989 & 0.9048 & 0.9048 \\
\hline Abbadeni & 0.7643 & 0.8006 & 0.7820 & 0.6256 & Amadasun & 0.7771 & 0.7248 & 0.7500 & 0.7500 \\
\hline Correlation & 0.6129 & 0.5948 & 0.6037 & 0.6037 & Abbadeni & 0.8409 & 0.8223 & 0.8315 & 0.7484 \\
\hline SRE & 0.7009 & 0.8092 & 0.7512 & 0.6009 & Haralick & 0.7231 & 0.6710 & 0.6961 & 0.6265 \\
\hline LH & 0.6844 & 0.8041 & 0.7394 & $0.5915^{4}$ & \multirow{3}{*}{\multicolumn{5}{|c|}{ (b) }} \\
\hline DGD & 0.6809 & 0.7890 & 0.7310 & 0.5848 & & & & & \\
\hline FD & 0.6025 & 0.6821 & 0.6399 & 0.5759 & & & & & \\
\hline SNE & 0.6285 & 0.7423 & 0.6807 & 0.5446 & Directionality & ion & all & $f^{p}$ & $a^{p}$ \\
\hline Tamura & 0.5497 & 0.5600 & 0.5548 & 0.4993 & measure & ion & ecall & $J_{k}^{k}$ & $g_{k}$ \\
\hline Weszka & 0.5424 & 0.5652 & 0.5536 & 0.4982 & Tamura & 0.7972 & 0.7636 & 0.7800 & 0.7800 \\
\hline ED & 0.5031 & 0.5245 & 0.5135 & 0.4622 & Abbadeni & 0.8146 & 0.8192 & 0.8169 & 0.7361 \\
\hline Contrast & 0.4776 & 0.5235 & 0.4995 & 0.4496 & Fourier & 0.6715 & 0.6633 & 0.6674 & 0.6674 \\
\hline
\end{tabular}

(a)

(c)

$$
a_{k, i}^{p}=c_{k, i-1}^{p} \quad d_{k, i}^{p}=b_{k, i+1}^{p}
$$
$\infty$.

In addition, it should be noticed that $a_{k, 1}^{p}=b_{k, 1}^{p}=-\infty$ and $c_{k, N_{k}^{p}}^{p}=d_{k, N_{k}^{p}}^{p}=$

At this point, once a fuzzy partition has been defined for each computational measure, the aim is to obtain a goodness measure that allows us to compare the ability of each partition to model the corresponding texture property. In our approach, given a partition $\Pi_{k}^{p}$, we propose to define a goodness measure $g_{k}^{p}$ associated to it as:

$$
g_{k}^{p}=f_{k}^{p} \cdot h_{k}^{p}
$$

This goodness measure will take into account two factors:

- $f_{k}^{p} \in[0,1]$, that measures whether the results given by the partition are in accordance with what a human would expect.

- $h_{k}^{p} \in[0,1]$, which is a factor that will depend on the number of classes $N_{k}^{p}$ that can be discriminated by the measure.

Thus, the highest goodness will be obtained for the partition that maximizes the product of both factors, i.e. a good partition must be able to discriminate as many different classes of the property as possible providing results that are in accordance with what a human would expect. In order to evaluate the relationship between the results given by $\Pi_{k}^{p}$ and the human perception, a test using the sub-images in $\mathcal{I}_{\mathcal{W}}^{p, t e s t}$ will be applied. This test will study whether the class in which each sub-image is classified according to the poll matches the corresponding class in the proposed partition. The metrics precision and recall will be used, as will be shown below.

Given an image $I_{i, w}^{p} \in \mathcal{I}_{\mathcal{W}}^{p, t e s t}$, first we will identify the initial class $C_{j} \in$ $\operatorname{Part}_{\mathcal{I}_{\mathcal{W}}^{p}}, j=1, \ldots, R^{p}$ in which this image has been classified according to 
the poll. Then, we will obtain the class $C_{k, n}^{p} \in \Upsilon_{k}^{p}, n=1, \ldots, N_{k}^{p}$ in which Algorithm 1 has grouped the initial class $C_{j}$. Thus, the test with the image $I_{i, w}^{p}$ will be considered as a success if $m_{i, w}^{p, k} \in\left(T_{k, n}^{p}\right)_{\alpha}$, with $m_{i, w}^{p, k}$ being the result of applying the measure $F_{k}^{p}$ to the sub-image $I_{i, w}^{p}$, and with $\left(T_{k, n}^{p}\right)_{\alpha}$ being the $\alpha$-cut at level $\alpha$ of $T_{k, n}^{p}$ (i.e. $\left(T_{k, n}^{p}\right)_{\alpha}=\left\{x \mid T_{k, n}^{p}(x) \geq \alpha\right\}$ ), where $T_{k, n}^{p}$ is the fuzzy set associated to the class $C_{k, n}^{p}$. The test will be considered as a failure otherwise. In this paper, we have considered the value $\alpha=0.2$ in order to avoid the outliers of each class. This implies that, for a test image, the membership degree to the corresponding fuzzy set must be at least 0.2 to be considered as a success.

We shall use the metrics precision and recall, widely used in classification tasks, for assessing the goodness of the proposed partitions. Let true_positives $\left(C_{k, i}^{p}\right)$, false_positives $\left(C_{k, i}^{p}\right)$ and false_negatives $\left(C_{k, i}^{p}\right)$ be the true positives, false positives and false negatives for the class $C_{k, i}^{p}$, respectively. The metrics precision and recall can be computed for each class $C_{k, i}^{p} \in \Upsilon_{k}^{p}$ as:

$\left.\operatorname{precision}\left(C_{k, i}^{p}\right)=\frac{\text { true_positives }}{(} C_{k, i}^{p}\right)$ true_positives $\left(C_{k, i}^{p}\right)+$ false_positives $\left(C_{k, i}^{p}\right)$

$\left.\operatorname{recall}\left(C_{k, i}^{p}\right)=\frac{\text { true_positives }}{(} C_{k, i}^{p}\right)$ true_positives $\left(C_{k, i}^{p}\right)+\operatorname{false\_ negatives}\left(C_{k, i}^{p}\right)$

The global metrics precision $_{k}^{p}$ and recall ${ }_{k}^{p}$ for the measure $F_{k}^{p}$ associated to the partition $\Pi_{k}^{p}$ will be computed as the average of the corresponding metrics for all the classes $C_{k, i}^{p} \in \Upsilon_{k}^{p}, i=1, \ldots, N_{k}^{p}$. The second and third columns of tables 2(a), 2(b) and 2(c) show the obtained metrics for the proposed fuzzy partitions corresponding to the properties of fineness, contrast and directionality, respectively.

In order to give a single value to evaluate the performance of each partition, it is usual to combine both metrics into a single measure called $f$-measure or balanced f-score, defined as [74]:

$$
f_{k}^{p}=2 \cdot \frac{\text { precision }_{k}^{p} \cdot \text { recall }_{k}^{p}}{\text { precision }_{k}^{p}+\text { recall }_{k}^{p}}
$$

The obtained $f_{k}^{p}$ value for each fuzzy partition is shown in the fourth column of tables 2(a), 2(b) and 2(c). Thus, this value $f_{k}^{p}$ will be used in $g_{k}^{p}$ to measure the relationship between the results given by $\Pi_{k}^{p}$ and the human perception of the property. The other component considered in $g_{k}^{p}$, noted as $h_{k}^{p}$, will take into account the number of classes $N_{k}^{p}$ that can be discriminated by the measure, and we propose to define it as follows: 


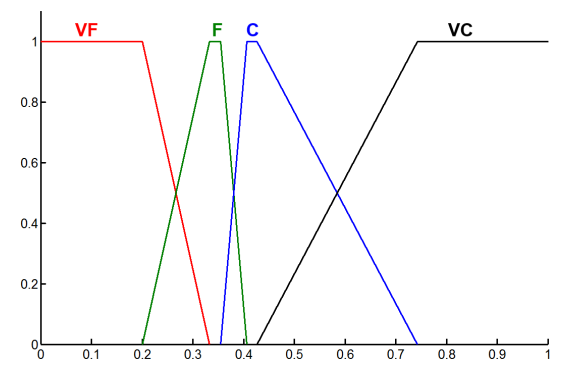

\begin{tabular}{ccccc}
\multicolumn{6}{c}{ Fineness (Amadasun) } \\
\hline $\mathrm{i}$ & $a_{k}^{i}$ & $b_{k}^{i}$ & $c_{k}^{i}$ & $d_{k}^{i}$ \\
\hline 1 & $-\infty$ & $-\infty$ & 0.2008 & 0.3326 \\
2 & 0.2008 & 0.3326 & 0.3551 & 0.4062 \\
3 & 0.3551 & 0.4062 & 0.4267 & 0.7430 \\
4 & 0.4267 & 0.7430 & $\infty$ & $\infty$ \\
\hline
\end{tabular}

Linguistic labels:

VF: "very fine", F: "fine", C: "coarse", VC: "very coarse"

(a)

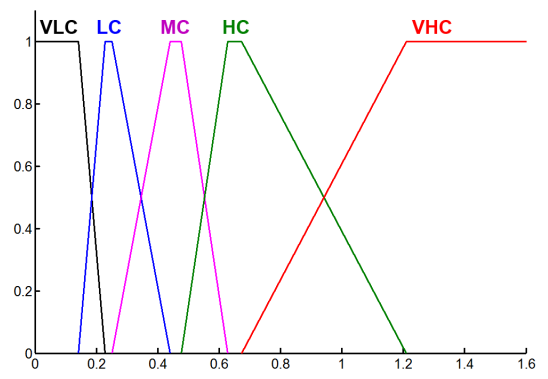

\begin{tabular}{ccccc}
\multicolumn{5}{c}{ Contrast (Tamura) } \\
\hline $\mathrm{i}$ & $a_{k}^{2}$ & $b_{k}^{i}$ & $c_{k}^{2}$ & $d_{k}^{2}$ \\
\hline 1 & $-\infty$ & $-\infty$ & 0.1410 & 0.2289 \\
2 & 0.1410 & 0.2289 & 0.2507 & 0.4405 \\
3 & 0.2507 & 0.4405 & 0.4774 & 0.6282 \\
4 & 0.4774 & 0.6282 & 0.6733 & 1.2093 \\
5 & 0.6733 & 1.2093 & $\infty$ & $\infty$ \\
\hline
\end{tabular}

Linguistic labels:

VLC: "very low contrasted", LC: "low contrasted", MC "medium contrasted", HC: "high contrasted", VHC: "very high contrasted"

(b)

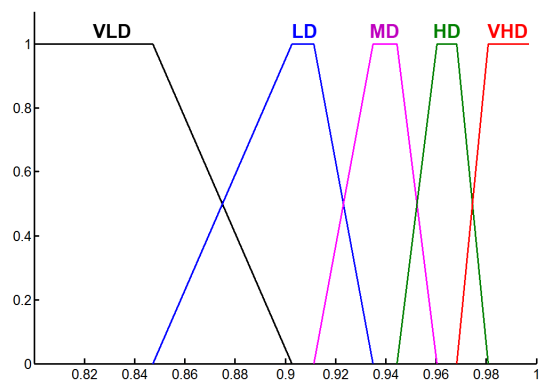

\begin{tabular}{ccccc}
\multicolumn{5}{c}{ Directionality (Tamura) } \\
\hline $\mathrm{i}$ & $a_{k}^{i}$ & $b_{k}^{i}$ & $c_{k}^{i}$ & $d_{k}^{i}$ \\
\hline 1 & $-\infty$ & $-\infty$ & 0.8471 & 0.9027 \\
2 & 0.8471 & 0.9027 & 0.9113 & 0.9350 \\
3 & 0.9113 & 0.9350 & 0.9445 & 0.9604 \\
4 & 0.9445 & 0.9604 & 0.9682 & 0.9807 \\
5 & 0.9682 & 0.9807 & $\infty$ & $\infty$ \\
\hline
\end{tabular}

Linguistic labels:

VLD: "very low directional", LD: "low directional", MD: "medium directional", HD: "high directional", VHD: "very high directional"

(c)

Figure 4: Proposed fuzzy partitions $\Pi_{k}^{p}$ corresponding to the properties of fineness (a), contrast (b) and directionality (c). 


$$
h_{k}^{p}= \begin{cases}1 & \text { if } N_{k}^{p}=M A X_{N_{k}^{p}} \\ 1-\rho \cdot\left(M A X_{N_{k}^{p}}-N_{k}^{p}\right) & \text { if } 1<N_{k}^{p}<M A X_{N_{k}^{p}} \\ 0 & \text { if } N_{k}^{p}=1\end{cases}
$$

with $M A X_{N_{k}^{p}}=\max \left\{N_{k}^{p}, k=1, \ldots, K_{p}\right\}$. Thus, the maximum value of this component, which is $h_{k}^{p}=1$, will correspond to the partitions with the maximum number of fuzzy sets among all the analyzed partitions (in this paper $M A X_{N_{k}^{p}}=$ $5)$. The value of $h_{k}^{p}$ will be reduced by a factor $\rho$ as $N_{k}^{p}$ decreases, and it will be 0 if $N_{k}^{p}=1$, since in this case the partition contains a unique fuzzy set, which is not useful. In our approach, a factor $\rho=0.1$ has been considered, so $h_{k}^{p}=1$ for $N_{k}^{p}=5, h_{k}^{p}=0.9$ for $N_{k}^{p}=4$ and $h_{k}^{p}=0.8$ for $N_{k}^{p}=3$ (there are no partitions with $\left.N_{k}^{p} \leq 3\right)$.

The fifth column of tables 2(a), 2(b) and 2(c) shows the goodness measure $g_{k}^{p}$ for the fuzzy partitions corresponding to the properties of fineness, contrast and directionality, respectively. Note that these tables have been sorted in decreasing order of this value. In our experiments, the fuzzy partitions with the highest goodness value are obtained by using the measure of Amadasun in the case of fineness, and the measures of Tamura in the case of contrast and directionality. The parameters corresponding to these fuzzy partitions, as well as their graphical representation, are shown in Figure 4.

Regarding the linguistic labels associated to each fuzzy set, we propose to combine the terms related to the texture property ('fine/coarse', 'contrasted', 'directional') with linguistic hedges such as 'very', 'medium', 'low', etc., that are usually employed by humans to describe the gradual presence of a given concept $[4,75]$. This way, linguistic labels such as 'very fine', 'medium contrasted' or 'very low directional' have been proposed, as it is shown in Figure 4.

Let us remark that, when applying our approach for different random choices of learning and test examples, the obtained parameters for the fuzzy partitions vary less than $0.5 \%$ of the size of the support of the label in all cases. Hence, our approach is robust in the estimation of the parameters. This was expected because of our use of average value and confidence interval for calculating the kernel of the linguistic labels.

\section{Results}

In this section, the goodness of the fuzzy sets proposed for fineness, contrast and directionality properties will be analyzed. First, in section 8.1, we will compare them with the state of the art in the fuzzy modelling of visual texture properties. Then, the proposed fuzzy sets will be applied to several examples with images in order to analyze their performance. In particular, the fuzzy partitions $\Pi_{k}^{p}$ with the highest goodness value (shown in Figure 4) will be employed. The results for fineness, contrast and directionality properties are shown in sections 8.2, 8.3 and 8.4, respectively. In addition, in section 8.5 we will show an example where the 
proposed fuzzy sets are applied for content-based image retrieval using linguistic labels.

\subsection{Comparison with the state of the art}

At this point, the aim is to compare the fuzzy sets proposed in this paper (the fuzzy partitions obtained in the previous section) with the state of the art in the fuzzy modelling of visual texture properties. As we have commented in section 2 , two different group of fuzzy approaches can be found in the literature. In the first group, each texture property is modeled as a whole by means of a unique fuzzy set defined on the domain of different computational measures, that directly represents the presence degree of the property [51, 52]. As only one label is used in this type of techniques (the label associated to the unique fuzzy set), in order to compare these techniques with our approach, we need a strategy to generate $N_{k}^{p}$ different labels. For this purpose, a crisp partition of $N_{k}^{p}$ equi-spaced intervals has been performed in the membership degree domain, with $N_{k}^{p}$ being the number of classes in the fuzzy partition that it is compared with. This way, each interval can be associated to the corresponding fuzzy set in the partition on the basis of its position in the membership degree domain.

The second group of fuzzy approaches in the literature is based on fuzzy partitions. The majority of these techniques $[10,54,4,55,56]$ propose a similar solution: for each texture property, a fuzzy partition with five linguistic terms is defined on the domain of the corresponding measure of Tamura. In these approaches, each fuzzy partition is generated through an unsupervised fuzzy clustering algorithm on the basis of the measure values obtained from a set of texture images. In order to include the fuzzy partitions proposed in those works in our comparative study, we have generated them by performing a fuzzy clustering on the subset of images $\mathcal{I}_{\mathcal{W}}^{p \text {,analysis }}$ commented in the previous sections. In this comparative study, the items "Unique fuzzy set" and "Fuzzy clustering" refer to the first and the second type of fuzzy approaches in the literature, respectively, while the item "Ours" refers to the fuzzy partition proposed in this paper.

The statistic $f_{k}^{p}$ proposed in equation (6), that measures whether the results given by the partition are in accordance with what a human would expect, has been employed in this comparative study. As it has been commented in section 7 , in order to evaluate the relationship between the fuzzy partitions and the human perception, we have to study whether the class in which each subimage is classified according to the poll matches the corresponding class using the fuzzy partition. Thus, in the case of the "Fuzzy clustering" approach, we have to associate the fuzzy sets in each partition with the classes obtained by applying the Algorithm 1 for the measure of Tamura. In a similar way, the intervals in the membership degree domain of the "Unique fuzzy set" approach should be associated with the classes obtained by applying this algorithm for the measure used as reference set.

If the number of classes proposed in the literature approach match with the corresponding number of classes obtained in the distinguishability analysis, we 
Table 3: Comparative study between the "Fuzzy clustering" approach ("FC (Tamura)"), the "Unique fuzzy set" approach using the measure of Tamura ("UFS (Tamura)") and using the measure with the highest goodness value ("UFS (best)"), our approach using the measure of Tamura ("Ours (Tamura)") and our approach using the measure with the highest goodness value ("Ours (best)").

\begin{tabular}{lccccccccc} 
& \multicolumn{3}{c}{ Fineness } & \multicolumn{3}{c}{ Contrast } & \multicolumn{3}{c}{ Directionality } \\
\cline { 2 - 10 } Fuzzy model & precision & recall & $f_{k}^{p}$ & precision & recall & $f_{k}^{p}$ & precision & recall & $f_{k}^{p}$ \\
\hline FC (Tamura) & 0.5766 & 0.5028 & 0.5372 & 0.5838 & 0.5216 & 0.5509 & 0.7869 & 0.7200 & 0.7520 \\
UFS (Tamura) & 0.5127 & 0.4780 & 0.4948 & 0.7156 & 0.6991 & 0.7072 & 0.6073 & 0.5922 & 0.5997 \\
UFS (best) & 0.5308 & 0.6213 & 0.5725 & 0.7156 & 0.6991 & 0.7072 & 0.6073 & 0.5922 & 0.5997 \\
Ours (Tamura) & 0.5497 & 0.5600 & 0.5548 & 0.9109 & 0.8989 & 0.9048 & 0.7972 & 0.7636 & 0.7800 \\
Ours (best) & 0.7486 & 0.7744 & 0.7613 & 0.9109 & 0.8989 & 0.9048 & 0.7972 & 0.7636 & 0.7800 \\
\hline
\end{tabular}

can establish a direct correspondence. This way, we can assume that each class in the partition of the literature approach is associated to the corresponding class $C_{k, n}^{p}$, where $p$ is the texture property, $k$ is the measure used as reference set, and $n$ is the order of the class in the partition ${ }^{4}$. If, on the contrary, the number of classes does not match, Algorithm 1 should be properly applied in order to obtain the number of classes proposed in the literature. For example, in the case of the fineness property, five linguistic labels have been proposed in the "Fuzzy clustering" approach, but, as it was commented in section 6, only four classes can be discriminated by the measure of Tamura. This way, we propose to apply Algorithm 1, but in this case the iterative grouping process is stopped when five classes are achieved, although they are not distinguishable.

Table 3 shows the results obtained in this comparative study for the properties of fineness, contrast and directionality according to the statistic $f_{k}^{p}$. We have compared the "Fuzzy clustering" approach ("Fuzzy clustering (Tamura)"), the approach based on a unique fuzzy set using the measure of Tamura ("Unique fuzzy set (Tamura)") and using the measure with the highest goodness value ("Unique fuzzy set (best)"), and our approach using the measure of Tamura ("Ours (Tamura)") and using the measure with the highest goodness value ("Ours (best)"). As it has been shown in section 7, in the case of contrast and directionality properties, the partition with the highest goodness value is obtained for the measure of Tamura, so "Ours (best)" and "Ours (Tamura)" correspond to the same fuzzy model. A similar situation has happened in the case of the "Unique fuzzy set" approach. We can see that, for both properties, a higher value of $f_{k}^{p}$ is obtained with our approach, which implies that the fuzzy partitions proposed in this paper for the measure of Tamura improves the partitions in the literature. In the case of the fineness property, our fuzzy partition for the measure of Tamura also improves the literature approaches, but, in ad-

\footnotetext{
${ }^{4}$ Note that each fuzzy set can be ordered semantically in a partition according to its linguistic label.
} 


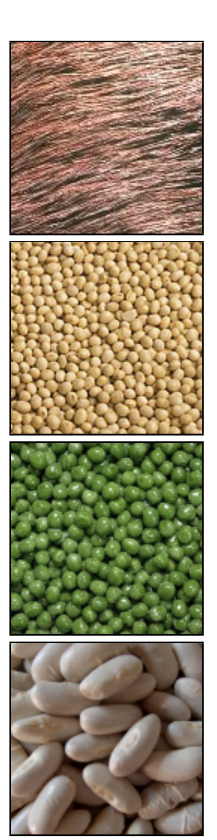

(a)

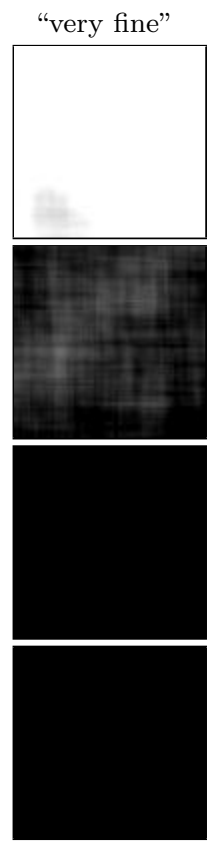

(b)
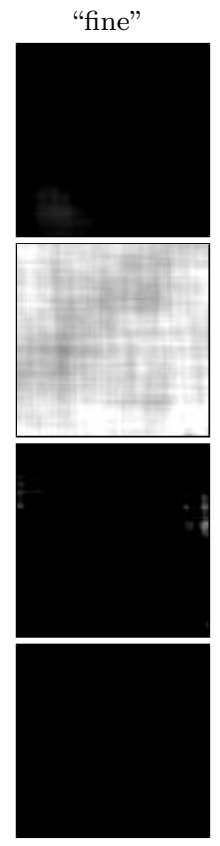

(c)
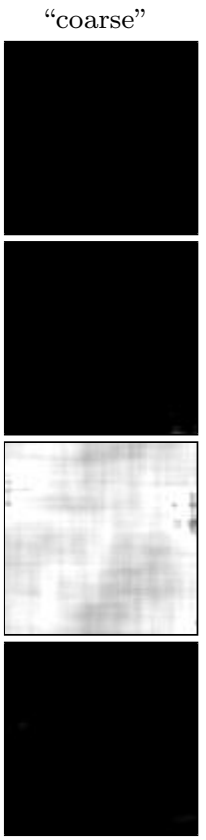

(d)

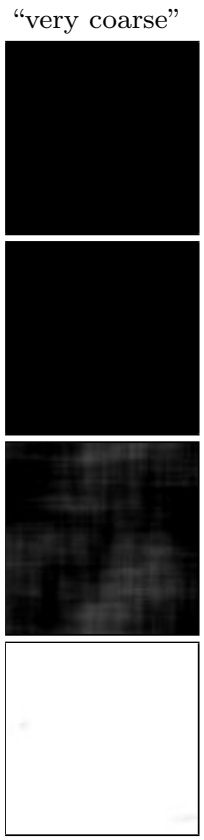

(e)

Figure 5: Result for a collection of texture images. (a) Original images. (b)(e) Mapping from the original images to the membership degree corresponding to the fuzzy sets with labels "very fine", "fine", "coarse" and "very coarse", respectively, obtained by applying the proposed fuzzy partition for the fineness property.

dition, we have proposed a fuzzy model using the measure of Amadasun that is more suitable to represent the fineness property.

When applying our approach for different random choices of learning and test examples, as commented in the previous section, the obtained parameters for the fuzzy partitions do not vary significantly, and consequently the comparison with other techniques shown in Table 3 remains the same, with differences in the statistic of less than 0.01 in all cases. Hence, our approach is also robust regarding comparison with other techniques.

In order to assess the ability of our fuzzy models to represent the presence degree of a texture property, the results obtained by applying these models to experiments with different images will be shown in next sections.

\subsection{Fineness results}

In this section, two experiments using the proposed fuzzy partition for the fineness property are shown. For the first one, we have considered Figure 5(a), cor- 


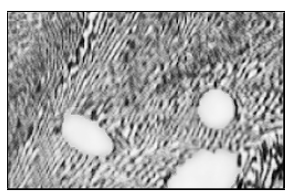

(a)

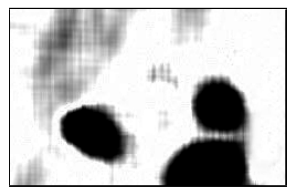

(e)

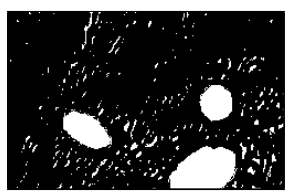

(b)

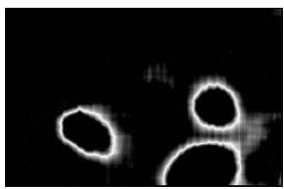

(f)

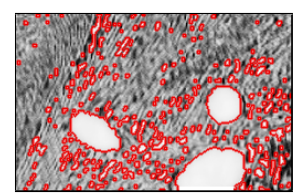

(c)

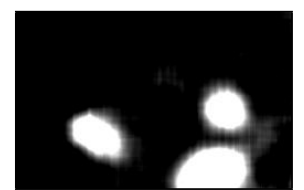

(g)

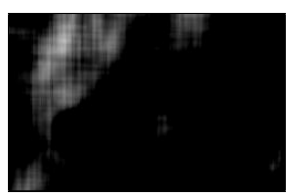

(d)

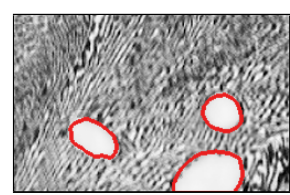

(h)

Figure 6: Pattern recognition. (a) Original image (b) Binary image obtained by thresholding the original one. (c) Region outlines of $b$ superimposed on the original image. (d)-(g) Mapping from the original image to the membership degree corresponding to the fuzzy sets "very fine", "fine", "coarse" and "very coarse", respectively. (h) Region outlines obtained by thresholding $g$ ("very coarse" texture) superimposed on the original image.

responding to a collection of texture images, each one with a different decreasing perception degree of fineness. Figures 5(b)-(e) show a mapping from the original texture images to the membership degree corresponding to the fuzzy sets with labels "very fine", "fine", "coarse" and "very coarse", respectively, obtained by applying the proposed fuzzy partition. In order to obtain these mappings, for each pixel in the original images, a centered window of size $32 \times 32$ has been analyzed and its membership degree to each fuzzy set has been calculated. This degree have been mapped into a gray level from 0 to 255. Thus, for example, Figure 5(b) represents the degree in which the texture is perceived as "very fine", with a white level meaning degree 1 , and a dark one meaning degree 0 . It can be noticed that our model captures the evolution of the perception degrees of fineness, and the obtained mappings can be directly interpreted by humans.

The second experiment, where the proposed fineness model has been employed for pattern recognition, is presented in Figure 6. In this case, the microscopy image shown in Figure 6(a), corresponding to the microstructure of a metal sample, has been used. The lamellae indicates islands of eutectic, which are to be separated from the uniform light regions. The brightness values in regions of the original image are not distinct, so texture information is needed for extracting the uniform areas. This fact is showed in figures 6(b) and 6(c), where a thresholding on the original image is displayed (homogeneous regions cannot be separated from the textured ones as they "share" brightness values).

As in the previous experiment, the proposed fuzzy partition is used to obtain the membership degree to the fuzzy sets with labels "very fine", "fine", "coarse" and "very coarse", and the corresponding mappings are shown in figures 6(d)- 
(g), respectively. It can be noticed that uniform light regions correspond to areas with high membership degrees to the fuzzy set "very coarse" (Figure 6(g)), and low membership degrees to the others. Thus, these regions can be extracted with ease by selecting the pixels of Figure $6(\mathrm{~g})$ with a degree higher than 0.9 (i.e. an $\alpha$-cut of the fuzzy set "very coarse" with $\alpha=0.9$ ), as it is shown in Figure 6(h). In addition, we can conclude from the obtained mappings that the eutectic mixture surrounding these regions is mostly a "fine" texture (Figure $6(\mathrm{e}))$.

Note that, if there are neighbouring regions in the image with a fineness presence corresponding to non-adjacent fuzzy sets, the boundary between these regions will appear in the mappings associated to the intermediate fuzzy sets, as can be seen in Figure 6(f). This happens because in the mapping process, windows that contain both textures are analyzed for pixels near the boundary, so the fineness measure gives an intermediate value.

\subsection{Contrast results}

In this section, two experiments using the proposed fuzzy partition for the contrast property are performed. For the first one, we have considered the collection of texture images shown in Figure 7(a), each one with a different decreasing perception degree of contrast. Figures 7(b)-(f) show a mapping from the original images to the membership degree corresponding to the fuzzy sets with labels "very high contrasted", "high contrasted", "medium contrasted", "low contrasted" and "very low contrasted", respectively. These mappings are obtained in a similar way to the fineness ones, by applying the proposed fuzzy partition for the contrast property. Thus, for example, Figure 7(b) represents the degree to which the texture is perceived as "very high contrasted", with a white level meaning maximum degree, and a dark one meaning zero degree. It can be noticed that the result obtained with our model matches what a human would expect, capturing the evolution of the perception degrees of contrast.

For the second experiment, let us consider Figure 8(a), corresponding to a natural image where several textures with different perception degrees of contrast are present. As in the previous experiment, the proposed fuzzy partition is used to obtain the membership degree to the fuzzy sets with labels "very high contrasted", "high contrasted", "medium contrasted", "low contrasted" and "very low contrasted", and the corresponding mappings are shown in figures 8(b)-(f), respectively. It can be noticed that, as it was expected, the region corresponding to the leopard skin has high membership degrees to the fuzzy set "very high contrasted" (Figure 8(b)), and very low for the rest. In addition, we can conclude that the branch of the tree can be considered as a low contrasted texture (Figure $8(\mathrm{e})$ ), and that the background is very low contrasted (Figure $8(\mathrm{f}))$.

Note that the boundary between different textures appears in the mappings associated to several fuzzy sets (figures 8(c)-(e)). This happens because in the mapping process, windows that contain both textures are analyzed for pixels near the boundary, so the value given by the contrast measure depends on the 


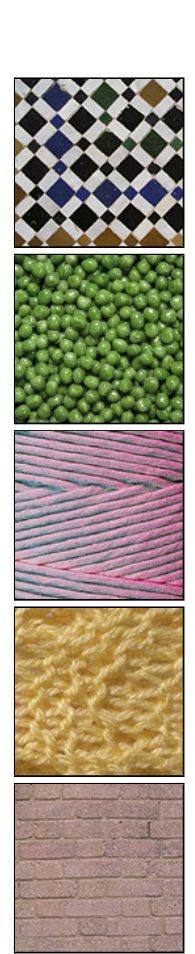

(a)

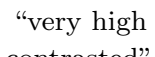

contrasted"
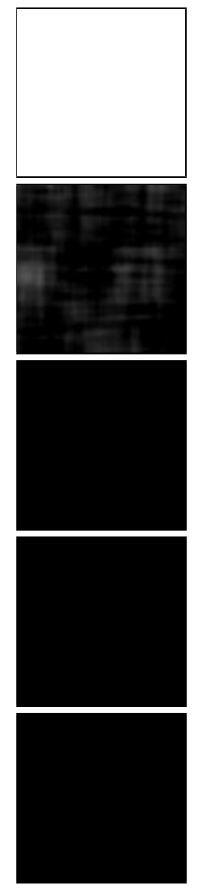

(b)
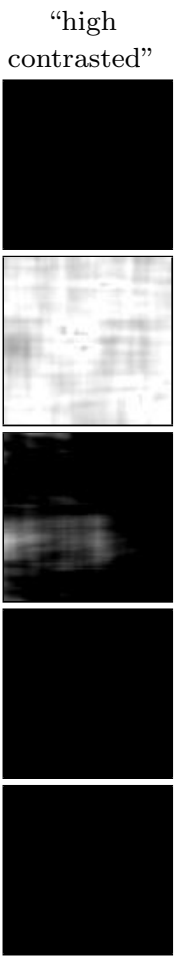

(c)
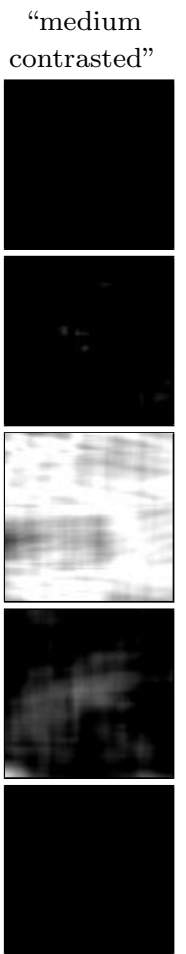

(d)
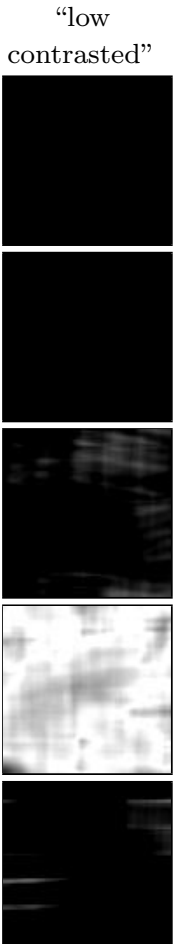

(e) "very low contrasted"
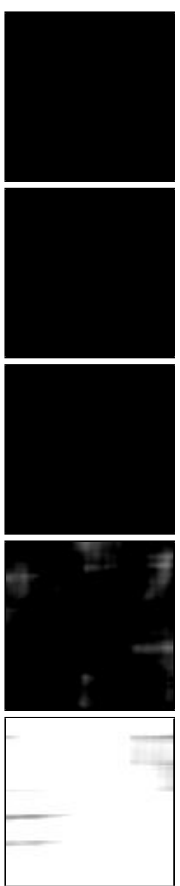

(f)

Figure 7: Result for a collection of texture images. (a) Original images. (b)-(f) Mapping from the original image to the membership degree corresponding to the fuzzy sets with labels "very high contrasted", "high contrasted", "medium contrasted", "low contrasted" and "very low contrasted", respectively, obtained by applying the proposed fuzzy partition for the contrast property.

contrast of each texture separately, as well as the contrast between them.

\subsection{Directionality results}

In the case of directionality, two experiments using the proposed fuzzy partition are also shown. For the first one, we have considered the collection of texture images shown in Figure 9(a), each one with a different decreasing perception degree of directionality. Figures 9(b)-(f) show a mapping from the original images to the membership degree corresponding to the fuzzy sets with labels "very high directional", "high directional", "medium directional", "low directional" and "very low directional", respectively. These mappings are obtained in a similar way to the previous ones, by applying the proposed fuzzy partition for the directionality property. It can be noticed that the proposed fuzzy partition 


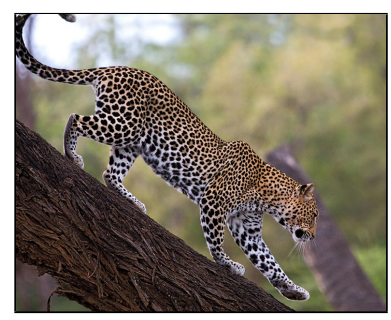

(a)

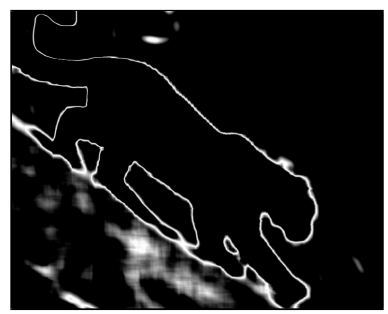

(d)

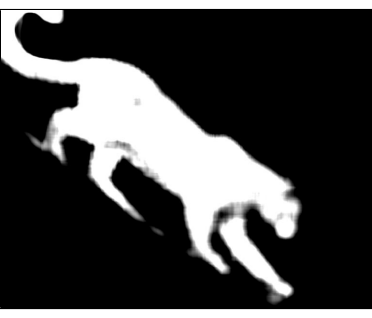

(b)

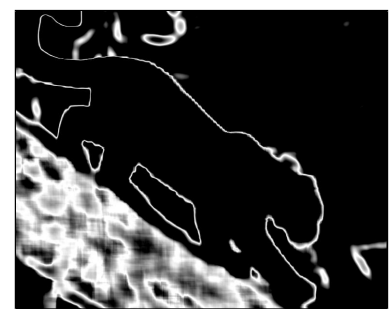

(e)

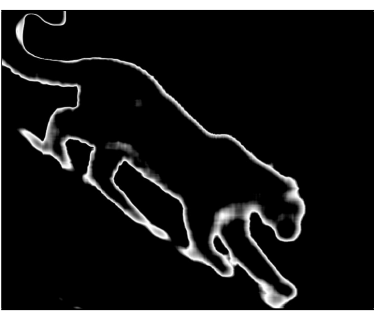

(c)

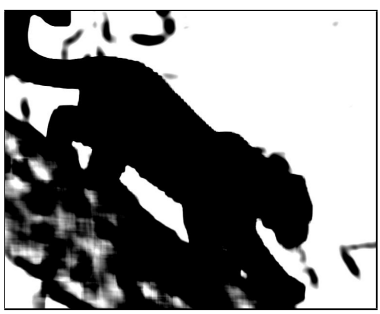

(f)

Figure 8: Result for a natural image. (a) Original image. (b)-(f) Mapping from the original image to the membership degree corresponding to the fuzzy sets with labels "very high contrasted", "high contrasted", "medium contrasted", "low contrasted" and "very low contrasted", respectively.

captures the evolution of the perception degrees of directionality.

For the second experiment, Figure 10(a) has been considered, corresponding to a natural image where several textures with different perception degrees of directionality are present. The proposed fuzzy partition is used to obtain the membership degree to the fuzzy sets "very high directional", "high directional", "medium directional", "low directional" and "very low directional", and the corresponding mappings are shown in figures 10(b)-(f). As can be seen, these mappings represents the directionality of the different textures in the image: the region corresponding to the door has high membership degrees to the fuzzy set "very high directional" (Figure 10(b)), and very low for the rest; the region of the brick wall has high membership degrees to the fuzzy set "low directional" (Figure 10(e)), while the region corresponding to the vine on the right side of the image can be considered as a very low directional texture (Figure 10(f)). Therefore, we can say that the obtained mappings are directly interpretable by humans.

Note that pixels near the boundary of adjacent textures have high membership degrees to the fuzzy set "very low directional" (Figure 9(f)). This happens because each texture has a different orientation, so windows that contain both textures in the mapping process are considered as non-directional textures. The boundary between different textures also appears in the mappings associated to the intermediate fuzzy sets (figures $9(\mathrm{c})-(\mathrm{e})$ ) as the directionality decreases 


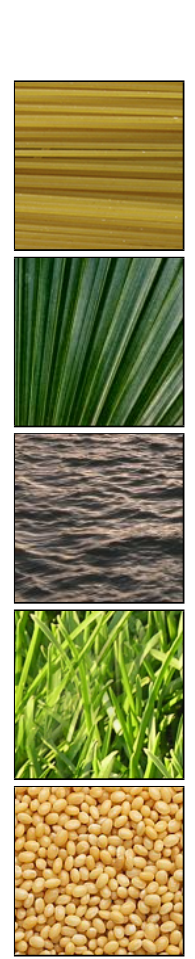

(a)

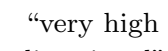

directional"
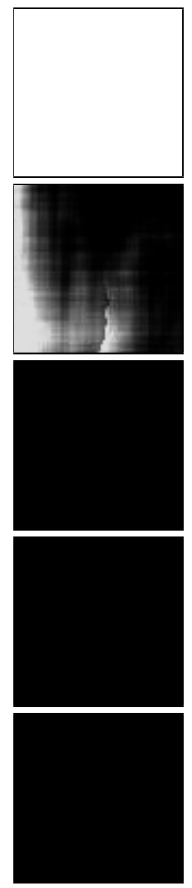

(b)
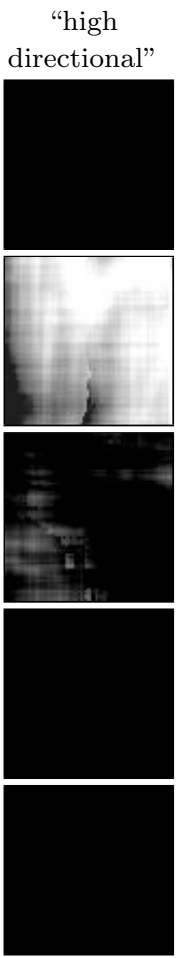

(c)
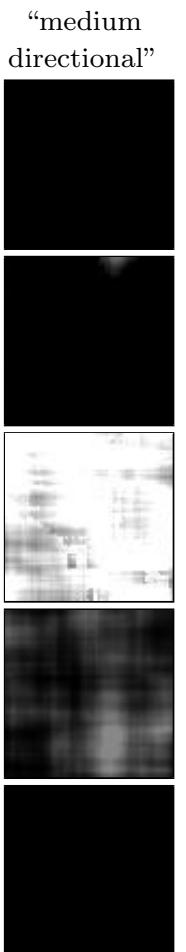

(d)
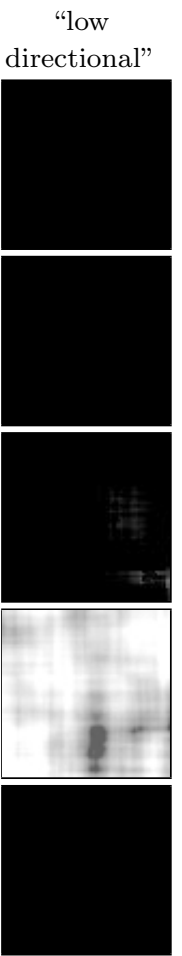

(e) "very low directional"
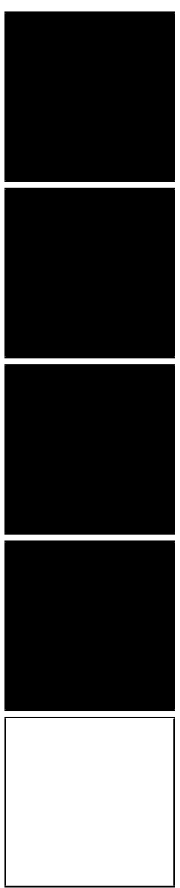

(f)

Figure 9: Result for a collection of texture images. (a) Original images. (b)-(f) Mapping from the original image to the membership degree corresponding to the fuzzy sets with labels "very high directional", "high directional", "medium directional", "low directional" and "very low directional", respectively, obtained by applying the proposed fuzzy partition for the directionality property.

gradually. This phenomenon will always appear, except in the case of adjacent textures with exactly the same orientation.

\subsection{Content-based image retrieval example}

In this section, the fuzzy partitions proposed in this paper are used for preliminary results on content-based image retrieval. In classical image retrieval systems, images are represented by vectors of features, and queries need to be defined as an image or sketch. In our approach, however, the proposed fuzzy sets can be used to describe images in terms of their texture fineness, contrast and directionality, allowing to perform queries on the basis of linguistic terms.

For this purpose, fuzzy dominant descriptors have been defined for each texture property in a similar way as the descriptors introduced in [76]. Let 


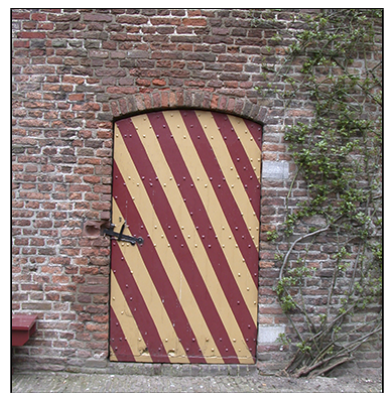

(a)

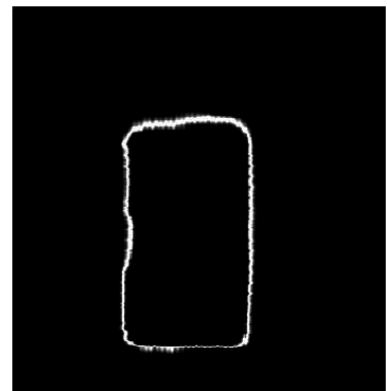

(d)

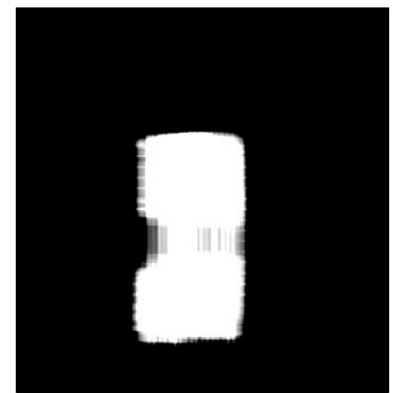

(b)

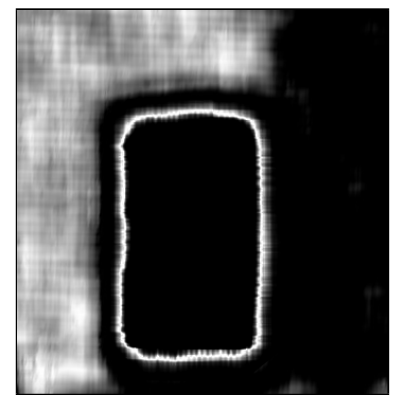

(e)

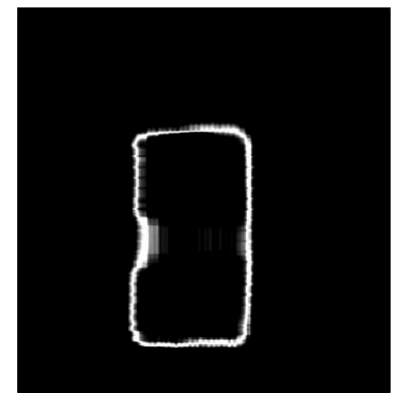

(c)

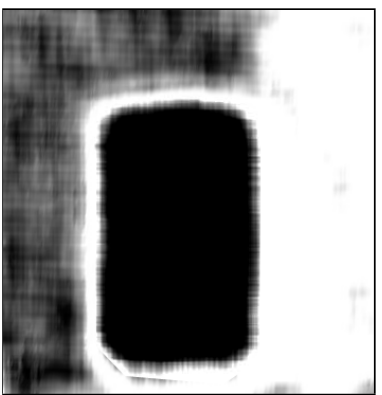

(f)

Figure 10: Result for a natural image. (a) Original image. (b)-(f) Mapping from the original image to the membership degree corresponding to the fuzzy sets with labels "very high directional", "high directional", "medium directional", "low directional" and "very low directional", respectively. 


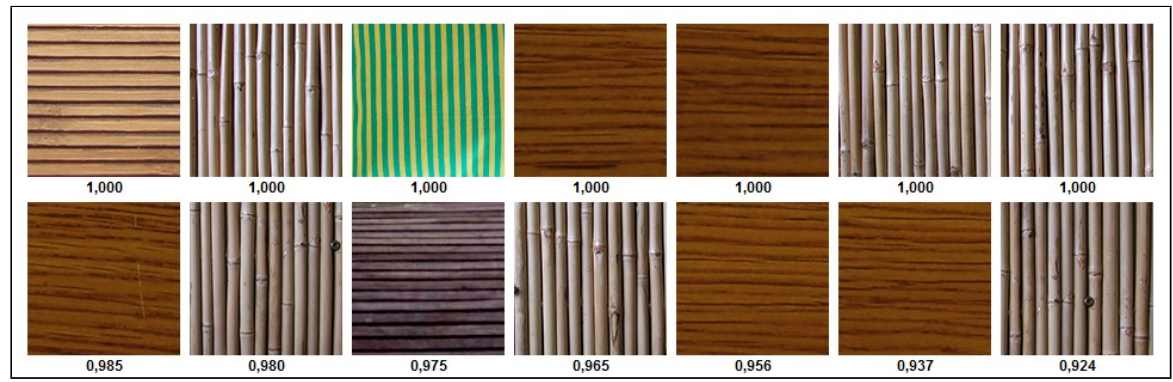

(a)

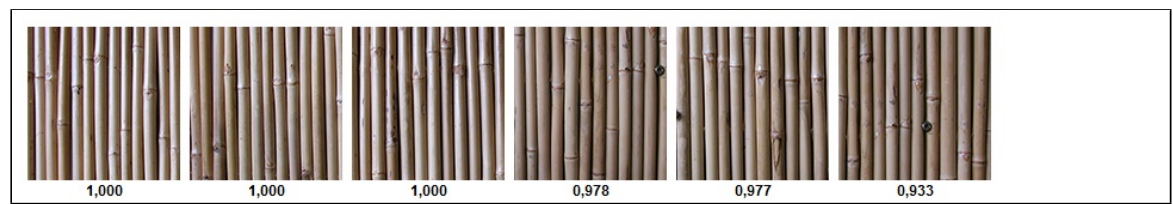

(b)

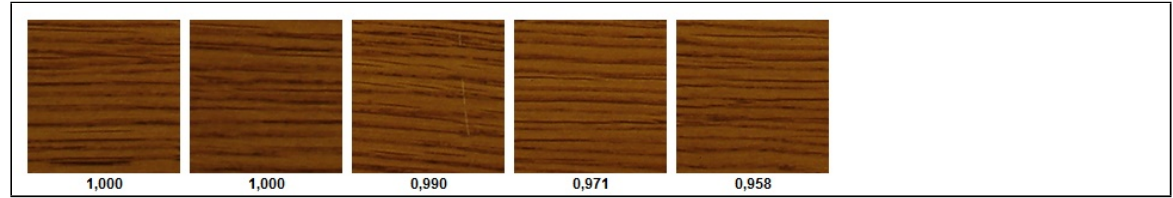

(c)

Figure 11: Retrieval results for the inclusion query with the labels Very high directional and Coarse (a), Very high directional, Coarse and Very high contrasted (b), and Very high directional, Coarse and Very low contrasted (c). 
$P=\mathscr{T}$ a finite reference universe of texture fuzzy sets which model fineness, directionality and contrast properties. We define the Fuzzy Dominant Texture Descriptor as the fuzzy set

$$
F D_{T D}=\sum_{T \in \mathscr{T}} \operatorname{Dom}(T) / T
$$

with $\operatorname{Dom}(T)$ being the dominance degree of a texture property $T$ as it is defined in [76]. For example, a Fuzzy Dominant Texture Descriptor $F D_{T D}=1.0 /$ fine $+0.8 /$ VeryDirectional $+0.2 /$ MediumContrast could be interpreted as the texture properties fine dominates with degree 1, VeryDirectional with degree 0.8 and MediumContrast with degree 0.2.

Moreover, fuzzy operators over this descriptors are proposed to define conditions in image retrieval queries. In this example, the fuzzy inclusion operator proposed in [77] is employed. Let $F D^{i}$ and $F D^{j}$ be two fuzzy descriptors defined over a finite reference universe of fuzzy sets $P$, let $S$ be a similarity relation defined over the elements of $P, \otimes$ a t-norm, and $J$ a fuzzy implication operator. The inclusion degree of $F D^{i}$ in $F D^{j}$ driven by the similarity relation $S$, the t-norm $\otimes$ and the fuzzy implication operator $J$ is calculated as follows:

$$
\Theta_{S, \otimes, J}\left(F D^{j}, F D^{i}\right)=\min _{\widetilde{x} \in P} \max _{\widetilde{y} \in P} \theta_{S, \otimes, J}^{i, j}(\widetilde{x}, \widetilde{y})
$$

where

$$
\theta_{S, \otimes, J}^{i, j}(\widetilde{x}, \widetilde{y})=\otimes\left(J\left(F D^{i}(\widetilde{x}), F D^{j}(\widetilde{y})\right), S(\widetilde{x}, \widetilde{y})\right)
$$

In this proposal, the minimum as t-norm $\otimes$, the similarity relation $S$ defined in equation 14 and the Lukasiewicz operator [78] as implication operator $J$ are used since by using them, equation (13) satisfies all the Sinha-Dougherty axioms for inclusion measures [79].

$$
S(\widetilde{x}, \widetilde{y})= \begin{cases}1 & \text { if } \widetilde{x}=\widetilde{y} \\ 0 & \text { otherwise }\end{cases}
$$

The database used in this retrieval system is an extension of the standard VisTex database [80], where new texture images have been incorporated in order to introduce more variety in the presence degree of the properties. In the first example, Figure 11(a) shows the retrieval results for the inclusion query with the linguistic labels Very high directional and Coarse (specifically, the query descriptor is $F D_{T D}^{q u e r y}=1 /$ veryHighDirectional $+1 /$ Coarse). Retrieved images are shown in decreasing order of the inclusion degree, that is shown below each image. Only the images with an inclusion degree higher than 0.9 are shown in this figure. It can be noticed that these images match the perception of very high directional and coarse textures.

More specific retrieval results can be obtained by adding contrast information, as it is shown in figures $11(\mathrm{~b})$ and $11(\mathrm{c})$. In the first case, the linguistic label Very high contrasted has been added to the query. As in Figure 11(a), only the images with an inclusion degree higher than 0.9 are shown. It can be noticed that "reeds" images are retrieved with the highest inclusion degree, 
because their textures are perceives as very high directional, coarse and very high contrasted. On the other hand, if the label Very low contrasted is added to query, "wood" images are retrieved with the highest inclusion degree, as it is shown in Figure 11(c).

\section{Conclusions and future works}

In this paper, a perception-based fuzzy approach for texture modelling has been proposed. In this approach, texture properties have been modelled by means of fuzzy partitions defined on the domain of computational measures of the corresponding property. Such solution achieves a double objective. On the one hand, fuzzy models that allow to represent the imprecision related to texture properties and to describe it in natural language have been obtained. In order to set the number of linguistic labels in the partition, the capability of each measure to discriminate between different presence degrees of the property has been studied, taking into account the relationship between the computational values given by the measures and the human perception of the corresponding property. This way, we ensure that the measure is able to provide significantly different values for all the proposed categories. The parameters of the membership functions have been also calculated using the information given by the distinguishability analysis, obtaining fuzzy partitions adapted to the human perception of the property. This way, the obtained results match what a human would expect, as shown in the experiments of section 8 .

On the other hand, the second objective achieved in our approach is the identification of the most appropriate computational measure for each property. In the proposed modelling, some of the most representative measures in the literature have been employed. We have concluded that, from all the measures used in this paper, the measure of Amadasun in the case of fineness, and the measures of Tamura for contrast and directionality, are the most suitable ones to represent the corresponding properties; moreover, the model that better fits each one is shown in Figure 4.

Regarding future works, as it was pointed out, the fuzzy sets proposed in this paper have been obtained by using the assessments given by a group of subjects in the polls, so they are representing the average perception of each property. Thus, these fuzzy sets can be considered as the default models for the corresponding properties, because they can be used if additional information is not available (user's profile, context, etc.). However, a particular user may have a different perception of these properties, and, moreover, the image context may influence the global perception. In order to take into account these considerations, an approach that allows to adapt the default models to each particular case is needed. In this sense, we are working on a methodology to automatically adapt the membership function associated to each fuzzy set according to the information given by an user to represent his particular perception, or the information about the context extracted from the textures present in the image.

In addition, as it was pointed out, in the fuzzy partitions proposed in this 
paper only one crisp measure is used as reference set, which implies that only the ability of this measure to capture the corresponding property is considered in the modelling. In some of our previous works [51], we have concluded that the combination of different computational measures improves the characterization of texture properties [51]. In this sense, we are working on a methodology to define the fuzzy partitions on the domain of a combination of several measures.

\section{Acknowledgements}

Part of this research was funded by the Spanish Ministry of Economy and Competitiveness and the European Regional Development Fund (FEDER) under project grant TIN2014-58227-P. We also would like to thank Dr. Daniel Sánchez for his valuable assistance in the field of fuzzy logic, as well as Elena GalánPerales for her contribution in the human assessment collection.

\section{References}

[1] J. C. Russ, The Image Processing Handbook, 3rd Edition, CRC Press and IEEE Press, 1999.

[2] A. R. Rao, G. L. Lohse, Identifying high level features of texture perception, Graphical Models and Image Processing 55 (3) (1993) 218-233.

[3] H. Tamura, S. Mori, T. Yamawaki, Textural features corresponding to visual perception, IEEE Transactions on Systems, Man and Cybernetics 8 (1978) 460-473.

[4] H. C. Lin, C. Y. Chiu, S. N. Yang, Finding textures by textual descriptions, visual examples, and relevance feedbacks, Pattern Recognition Letters 24 (14) (2003) 2255-2267.

[5] W. T. Lin, C. H. Lin, T. H. Wu, , Y. K. Chan, Image segmentation using the k-means algorithm for texture features, in: Proceedings of the International Conference on Computer, Electrical, and Systems Science, and Engineering, Venice, Italy, 2010, pp. 26-28.

[6] W. Niblack, R. Barber, W. Equitz, M. Flickner, E. H. Glasman, D. Petkovic, P. Yanker, C. Faloutsos, G. Taubin, The QBIC project: Querying images by content, using color, texture, and shape, in: Proceedings of Storage and Retrieval for Image and Video Databases, San Jose, CA, USA, 1993, pp. 173-187.

[7] S. Battiato, G. Gallo, S. Nicotra, Perceptive visual texture classification and retrieval, in: Proceedings of the 12th International Conference on Image Analysis and Processing, Washington, DC, USA, 2003, pp. 524-529. 
[8] B. S. Manjunath, P. Salembier, T. Sikora (Eds.), Introduction to MPEG7, Multimedia Content Description Interface, John Wiley and Sons, Ltd., 2002.

[9] M. Nirmala, K. Karthikeyan, S. Appalabatla, R. A. Ahmed, Image interpretation based on similarity measures of visual content descriptors. An insight, International Journal of Computer Science and Emerging Technologies 2 (2) (2011) 242-248.

[10] H. Aboulmagd, N. El-Gayar, H. Onsi, A new approach in content-based image retrieval using fuzzy, Telecommunication Systems 40 (1) (2009) 5566.

[11] M. Flicker, H. Sawhney, W. Niblack, J. Ashley, Q. Huang, B. Dom, M. Gorkani, J. Hafner, D. Lee, D. Petkovic, D. Steele, P. Yanker, Query by image and video content: the QBIC system, IEEE Computer 28 (9) (1995) $23-32$.

[12] Z. Huang, S. Lo, N. Mayr, W. Yuh, Texture segmentation in magnetic resonance images using discrete wavelet transform combined with gabor wavelets, Medical Physics 40 (2013) 175.

[13] S. Lahmiri, M. Boukadoum, Hybrid discrete wavelet transform and gabor filter banks processing for features extraction from biomedical images, Journal of Medical Engineering 2013 (2013) 1-13.

[14] S. Rahman, S. M. Naim, A. A. Farooq, M. Islam, Combination of Gabor and curvelet texture features for face recognition using principal component analysis, International Journal of Computer \& Electrical Engineering 4 (3) (2012) 264-269.

[15] F. Riaz, A. Hassan, S. Rehman, U. Qamar, Texture classification using rotation- and scale-invariant Gabor texture features, IEEE Signal Processing Letters 20 (6) (2013) 607-610.

[16] L. Zhang, Z. Zhou, H. Li, Binary Gabor pattern: An efficient and robust descriptor for texture classification, in: Proceedings of the 2012 IEEE International Conference on Image Processing, Orlando, FL, USA, 2012, pp. $81-84$.

[17] A. G. Zuñiga, J. B. Florindo, O. M. Bruno, Gabor wavelets combined with volumetric fractal dimension applied to texture analysis., Pattern Recognition Letters 36 (2014) 135-143.

[18] M. S. Allili, Wavelet modeling using finite mixtures of generalized gaussian distributions: Application to texture discrimination and retrieval, IEEE Transactions on Image Processing 21 (4) (2012) 1452-1464.

[19] Y. Dong, J. Ma, Wavelet-based image texture classification using local energy histograms, IEEE Signal Processing Letters 18 (4) (2011) 247-250. 
[20] S. Hu, C. Xu, W. Guan, Y. Tang, Y. Liu, Texture feature extraction based on wavelet transform and gray-level co-occurrence matrices applied to osteosarcoma diagnosis, Bio-Medical Materials and Engineering 24 (1) (2014) $129-143$.

[21] H. Ji, X. Yang, H. Ling, Y. Xu, Wavelet domain multifractal analysis for static and dynamic texture classification, IEEE Transactions on Image Processing 22 (1) (2013) 286-299.

[22] N. E. Lasmar, Y. Berthoumieu, Gaussian copula multivariate modeling for texture image retrieval using wavelet transforms, IEEE Transactions on Image Processing 23 (5) (2014) 2246-2261.

[23] Y. Qian, M. Ye, J. Zhou, Hyperspectral image classification based on structured sparse logistic regression and three-dimensional wavelet texture features, IEEE Transactions on Geoscience and Remote Sensing 51 (4) (2013) 2276-2291.

[24] D. Cheng, T. Sun, X. Jiang, A robust image classification scheme with sparse coding and multiple kernel learning, in: Digital Forensics and Watermaking, Vol. 7809 of Lecture Notes in Computer Science, Springer Berlin Heidelberg, 2013, pp. 520-529.

[25] J. Li, Y. Wang, S. Chu, J. F. Roddick, Kernel self-optimization learning for kernel-based feature extraction and recognition, Information Sciences 257 (2014) 70-80.

[26] D. Qin, X. Chen, M. Guillaumin, L. V. Gool, Quantized kernel learning for feature matching, in: Proceedings of the 2014 Conference on Neural Information Processing Systems, Quebec, Canada, 2014, pp. 172-180.

[27] A. Shrivastava, V. M. Patel, R. Chellappa, Multiple kernel learning for sparse representation-based classification, IEEE Transactions on Image Processing 23 (7) (2014) 3013-3024.

[28] D. Tuia, G. Camps-Valls, G. Matasci, M. Kanevski, Learning relevant image features with multiple-kernel classification, IEEE Transactions on Geoscience and Remote Sensing 48 (10) (2010) 3780-3791.

[29] L. Zhang, X. Zhen, L. Shao, Learning object-to-class kernels for scene classification., IEEE Transactions on Image Processing 23 (8) (2014) 3241-3253.

[30] Y. Gao, R. Ji, W. Liu, Q. Dai, G. Hua, Weakly supervised visual dictionary learning by harnessing image attributes, IEEE Transactions on Image Processing 23 (12) (2014) 5400-5411.

[31] S. Kong, D. Wang, A dictionary learning approach for classification: Separating the particularity and the commonality, in: Computer Vision - ECCV 2012, Vol. 7572 of Lecture Notes in Computer Science, Springer Berlin Heidelberg, 2012, pp. 186-199. 
[32] H. Wang, C. Yuan, W. Hu, C. Sun, Supervised class-specific dictionary learning for sparse modeling in action recognition, Pattern Recognition 45 (11) (2012) $3902-3911$.

[33] F. Zhu, L. Shao, Weakly-supervised cross-domain dictionary learning for visual recognition, International Journal of Computer Vision 109 (1-2) (2014) $42-59$.

[34] D. Atkins, K. Neshatian, M. Zhang, A domain independent genetic programming approach to automatic feature extraction for image classification, in: Proceedings of the 2011 IEEE Congress on Evolutionary Computation, 2011, pp. 238-245.

[35] C.-C. Lai, Y.-C. Chen, A user-oriented image retrieval system based on interactive genetic algorithm, IEEE Transactions on Instrumentation and Measurement 60 (10) (2011) 3318-3325.

[36] S. Li, H. Wu, D. Wan, J. Zhu, An effective feature selection method for hyperspectral image classification based on genetic algorithm and support vector machine, Knowledge-Based Systems 24 (1) (2011) 40-48.

[37] J. A. dos Santos, C. D. Ferreira, R. da S. Torres, M. A. Gonalves, R. A. C. Lamparelli, A relevance feedback method based on genetic programming for classification of remote sensing images, Information Sciences 181 (13) (2011) 2671-2684.

[38] L. Shao, L. Liu, X. Li, Feature learning for image classification via multiobjective genetic programming, IEEE Transactions on Neural Networks and Learning Systems 25 (7) (2014) 1359-1371.

[39] P. Srisook, K. Praditwong, Automatic feature weight assignment based on image retrieval using genetic algorithm, Advanced Materials Research 931932 (2014) 1402-1406.

[40] N. Abbadeni, Computational perceptual features for texture representation and retrieval, IEEE Transactions on Image Processing 20 (1) (2011) 236246.

[41] R. M. Haralick, Statistical and structural approaches to texture, Proceedings IEEE 67 (5) (1979) 786-804.

[42] P. N. Munje, D. Kapgate, S. Golai, Novel techniques for color and texture feature extraction, International Journal of Computer Science and Mobile Computing 3 (2) (2014) 497-507.

[43] N. Puviarasan, R. Bhavani, A. Vasanthi, Image retrieval using combination of texture and shape features, International Journal of Advanced Research in Computer and Communication Engineering 3 (3) (2014) 5873-5877. 
[44] G. Tamilpavai, S. T. Selvi, Multiple representations of perceptual features for texture classification and retrieval, International Journal of Computer Applications 48 (20) (2012) 5-11.

[45] W. H. W. Yussof, H. Burkhardt, Relational features for texture classification, in: Signal Processing, Image Processing and Pattern Recognition, Vol. 260 of Communications in Computer and Information Science, Springer Berlin Heidelberg, 2011, pp. 438-447.

[46] I. Bloch, A. Ralescu, Directional relative position between objects in image processing: a comparison between fuzzy approaches, Pattern Recognition 36 (7) (2003) 1563-1582.

[47] L. Khodja, L. Foulloy, E. Benoit, Fuzzy clustering for color recognition application to image understanding, in: Proceedings of the Fifth IEEE International Conference on Fuzzy Systems, Vol. 2, 1996, pp. 1407-1413.

[48] P. Matsakis, L. Wendling, A new way to represent the relative position between areal objects, IEEE Transactions on Pattern Analysis and Machine Intelligence 21 (7) (1999) 634-643.

[49] K. Miyajima, A. Ralescu, Fuzzy logic approach to model-based image analysis, in: Model-Based Vision, Vol. 1827, 1993, pp. 145-156.

[50] A.-Z. Shih, The approach of using fractal dimension and linguistic descriptors in CBIR, in: Proceedings of the 2012 International Conference on Machine Learning and Cybernetics, Vol. 5, Shaanxi, China, 2012, pp. $1704-1707$.

[51] J. Chamorro-Martínez, P. Martínez-Jiménez, J. Soto-Hidalgo, A. LeónSalas, A fuzzy approach for modelling visual texture properties, Information Sciences 313 (1) (2015) 1-21.

[52] J. Chamorro-Martínez, P. Martínez-Jiménez, J. Soto-Hidalgo, B. PradosSuárez, An adaptive fuzzy approach for modelling visual texture properties, Fuzzy Sets and Systems 286 (1) (2016) 86-113.

[53] D. Antonypandiarajan, K. Suganya, Performance analysis for coal texture classification, in: Proceedings of the 2012 International Conference on Emerging Trends in Science, Engineering and Technology, Tiruchirappalli, India, 2012, pp. 251-254.

[54] S. Kulkarni, B. Verma, Fuzzy logic based texture queries for CBIR, in: Proceedings of the 5th International Conference on Computational Intelligence and Multimedia Applications, Xi'an, China, 2003, pp. 223-228.

[55] S. S. Tripathy, R. Shekhar, R. S. Kumar, Texture retrieval system using intuitionistic fuzzy set theory, in: Proceedings of the 2011 International Conference on Devices and Communications, Jharkhand, India, 2011, pp. $1-5$. 
[56] B. Verma, S. Kulkarni, A fuzzy-neural approach for interpretation and fusion of colour and texture features for CBIR systems, Applied Soft Computing 5 (1) (2004) 119-130.

[57] E. Ruspini, A new approach to clustering, Information and Control 15 (1969) 22-32.

[58] J. Chamorro-Martínez, P. Martínez-Jiménez, A comparative study of texture coarseness measures, in: Proceedings of the 16th IEEE International Conference on Image Processing, Cairo, Egypt, 2009, pp. 1337-1340.

[59] N. Abbadeni, N. Ziou, D. S. Wang, Autocovariance-based perceptual textural features corresponding to human visual perception, in: Proceedings of the 15th International Conference on Pattern Recognition, Vol. 3, Barcelona, Spain, 2000, pp. 901-904.

[60] B. B. Mandelbrot, The fractal geometry of nature, Freeman, San Francisco, 1982.

[61] S. Peleg, J. Naor, R. Hartley, D. Avnir, Multiple resolution texture analysis and classification, IEEE Transactions on Pattern Analysis and Machine Intelligence 6 (4) (1984) 518-523.

[62] R. M. Haralick, K. Shanmugam, I. Dinstein, Textures features for image classification, IEEE Transactions on Systems, Man and Cybernetics 3 (1973) 610-621.

[63] M. Amadasun, R. King, Textural features corresponding to textural properties, IEEE Transactions on Systems, Man and Cybernetics 19 (5) (1989) $1264-1274$.

[64] M. M. Galloway, Texture analysis using gray level run lengths, Computer Graphics and Image Processing 4 (1975) 172-179.

[65] C. Sun, W. G. Wee, Neighboring gray level dependence matrix for texture classification, Computer Vision, Graphics and Image Processing 23 (1983) $341-352$.

[66] S. I. Kim, K. C. Choi, D. S. Lee, Texture classification using run difference matrix, in: Proceedings of the 1991 IEEE Ultrasonics Symposium, Vol. 2, Orlando, FL, USA, 1991, pp. 1097-1100.

[67] J. S. Weszka, C. R. Dyer, A. Rosenfeld, A comparative study of texture measures for terrain classification, IEEE Transactions on Systems, Man, and Cybernetics 6 (4) (1976) 269-285.

[68] S. D. Newsam, C. Kammath, Retrieval using texture features in high resolution multi-spectral satellite imagery, in: Proceedings of Data Mining and Knowledge Discovery: Theory, Tools, and Technology VI, Vol. 5433, Orlando, FL, USA, 2004, pp. 21-32. 
[69] H. Yoshida, D. D. Casalino, B. Keserci, A. Coskun, O. Ozturk, A. Savranlar, Wavelet-packet-based texture analysis for differentiation between benign and malignant liver tumours in ultrasound images, Physics in Medicine and Biology 48 (2003) 3735-3753.

[70] P. Brodatz, Textures: a photographic album for artists and designers, Dover Publishing Co., 1966.

[71] Y. Hochberg, A. Tamhane, Multiple Comparison Procedures, Wiley, 1987.

[72] P. Cohen, Empirical methods for artificial intelligence, MIT Press, Cambridge, MA, USA, 1995.

[73] D. Kleibaum, L. Kupper, K. Muller, Applied regression analysis and other multivariable methods, PWS Publishing Co., Boston, MA, USA, 1988.

[74] C. V. Rijsbergen, Information Retrieval, 2nd Edition, ButterworthHeinemann, Newton, MA, USA, 1979.

[75] Q. Li, Z. Shi, S. Luo, A neural network approach for bridging the semantic gap in texture image retrieval, in: Proceedings of the 2007 International Joint Conference on Neural Networks, 2007, pp. 581-585.

[76] J. Chamorro-Martínez, P. Martínez-Jiménez, J. Soto-Hidalgo, A fuzzy approach for retrieving images in databases using dominant color and texture descriptors, in: IEEE World Congress on Computational Intelligence (WCCI 2010), Barcelona (Spain), 2010, pp. 88-94.

[77] J. Chamorro-Martínez, J. Medina, C. Barranco, E. Galán-Perales, J. SotoHidalgo, Retrieving images in fuzzy object-relational databases using dominant color descriptors, Fuzzy Sets and Systems 158 (3) (2007) 312-324.

[78] C. Cornelis, C. Van der Donck, E. Kerre, Sinha-Dougherty approach to the fuzzification of set inclusion revisited, Fuzzy Sets and Systems 134 (2003) 283-295.

[79] D. Sinha, E. Dougherty, Fuzzification of set inclusion: Theory and applications, Fuzzy Sets and Systems 55 (1) (1993) 15 - 42. doi:http: //dx.doi.org/10.1016/0165-0114(93)90299-W.

[80] M. I. of Technology, Vistex: Vision texture database, http://vismod.media.mit.edu/vismod/imagery/VisionTexture/vistex.html. 